ESAIM: COCV 27 (2021) 46

https://doi.org/10.1051/cocv/2021049
ESAIM: Control, Optimisation and Calculus of Variations

www.esaim-cocv.org

\title{
LINEAR-QUADRATIC OPTIMAL CONTROL FOR BACKWARD STOCHASTIC DIFFERENTIAL EQUATIONS WITH RANDOM COEFFICIENTS
}

\author{
JingRUi SUn ${ }^{1, *}$ AND HANXIAO WANG ${ }^{2, * *, * * *}$
}

\begin{abstract}
This paper is concerned with a linear-quadratic (LQ, for short) optimal control problem for backward stochastic differential equations (BSDEs, for short), where the coefficients of the backward control system and the weighting matrices in the cost functional are allowed to be random. By a variational method, the optimality system, which is a coupled linear forward-backward stochastic differential equation (FBSDE, for short), is derived, and by a Hilbert space method, the unique solvability of the optimality system is obtained. In order to construct the optimal control, a new stochastic Riccati-type equation is introduced. It is proved that an adapted solution (possibly non-unique) to the Riccati equation exists and decouples the optimality system. With this solution, the optimal control is obtained in an explicit way.
\end{abstract}

Mathematics Subject Classification. 93E20, 49N10, 60H10.

Received March 11, 2020. Accepted May 3, 2021.

\section{INTRODUCTION}

Let $(\Omega, \mathcal{F}, \mathbb{P})$ be a complete probability space on which a standard one-dimensional Brownian motion $W=$ $\{W(t) ; t \geqslant 0\}$ is defined, and let $\mathbb{F}=\left\{\mathcal{F}_{t}\right\}_{t \geqslant 0}$ be the usual augmentation of the natural filtration generated by $W$. For a random variable $\xi$, we write $\xi \in \mathcal{F}_{t}$ if $\xi$ is $\mathcal{F}_{t}$-measurable, and for a process $\varphi$, we write $\varphi \in \mathbb{F}$ if it is $\mathbb{F}$-progressively measurable. For a matrix $H=\left(h_{i j}\right) \in \mathbb{R}^{k \times l}$, we use $|H|$ to denote the Frobenius norm of $H$, that is, $|H|=\left(\sum_{i, j}\left|h_{i j}\right|^{2}\right)^{\frac{1}{2}}$. Let $T>0$ be a fixed time horizon and $\mathbb{H}$ be a subset of $\mathbb{R}^{k \times l}$. For $t \in[0, T]$, we let

$$
\begin{aligned}
L_{\mathcal{F}_{t}}^{2}(\Omega ; \mathbb{H}) & =\left\{\xi: \Omega \rightarrow \mathbb{H} \mid \xi \in \mathcal{F}_{t} \text { and } \mathbb{E}|\xi|^{2}<\infty\right\}, \\
L_{\mathbb{F}}^{\infty}(t, T ; \mathbb{H}) & =\{\varphi:[t, T] \times \Omega \rightarrow \mathbb{H} \mid \varphi \in \mathbb{F} \text { and is bounded }\}
\end{aligned}
$$

\footnotetext{
*This author is supported by NSFC grant 11901280, Guangdong Basic and Applied Basic Research Foundation 2021A1515010031, and SUSTech start-up funds Y01286128 and Y01286228.

** This author is supported by Singapore MOE AcRF Grants R-146-000-271-112.

Keywords and phrases: Linear-quadratic optimal control, backward stochastic differential equation, random coefficient, stochastic Riccati equation.

1 Department of Mathematics, Southern University of Science and Technology, Shenzhen 518055, PR China.

2 Department of Mathematics, National University of Singapore, Singapore 119076, Singapore.

*** Corresponding author: hxwang14@fudan.edu.cn
} 
Consider the controlled linear backward stochastic differential equation (BSDE, for short):

$$
\left\{\begin{aligned}
\mathrm{d} Y(s) & =\{A(s) Y(s)+B(s) u(s)+C(s) Z(s)\} \mathrm{d} s+Z(s) \mathrm{d} W(s), \\
Y(T) & =\xi
\end{aligned}\right.
$$

where $A, C \in L_{\mathbb{F}}^{\infty}\left(0, T ; \mathbb{R}^{n \times n}\right)$ and $B \in L_{\mathbb{F}}^{\infty}\left(0, T ; \mathbb{R}^{n \times m}\right)$, called the coefficients of the state equation (1.1), are given processes; $u:[0, T] \times \Omega \rightarrow \mathbb{R}^{m}$, called a control process, is selected from a certain space to influence the state process $(Y, Z)$; and $\xi \in L_{\mathcal{F}_{T}}^{2}\left(\Omega ; \mathbb{R}^{n}\right)$, called an terminal state, is a given random variable. According to the standard result of BSDEs (see [25], for example), the state equation (1.1) admits a unique adapted solution $(Y, Z) \equiv\left(Y^{\xi, u}, Z^{\xi, u}\right)$ over $[t, T]$ whenever the control $u$ is square-integrable over $[t, T]$, i.e., whenever $u$ belongs to the following space:

$$
\mathcal{U}[t, T] \equiv L_{\mathbb{F}}^{2}\left(t, T ; \mathbb{R}^{m}\right)=\left\{\varphi:[t, T] \times \Omega \rightarrow \mathbb{R}^{m} \mid \varphi \in \mathbb{F} \text { and } \mathbb{E} \int_{t}^{T}|\varphi(s)|^{2} \mathrm{~d} s<\infty\right\}
$$

Moreover, there exists a constant $K>0$, independent of $(t, x)$ and $u$, such that

$$
\mathbb{E}\left[\sup _{t \leqslant s \leqslant T}|Y(s)|^{2}+\int_{t}^{T}|Z(s)|^{2} \mathrm{~d} s\right] \leqslant K \mathbb{E}\left[|\xi|^{2}+\int_{t}^{T}|u(s)|^{2} \mathrm{~d} s\right] .
$$

Let $\mathbb{S}^{n}$ be the set of symmetric $n \times n$ real matrices, and let $\mathbb{S}_{+}^{n}$ be the subset of $\mathbb{S}^{n}$ consisting of positive semidefinite matrices. To measure the performance of the control process $u$ over $[t, T]$, we introduce the following quadratic cost functional

$$
\begin{aligned}
J(t, \xi ; u)= & \mathbb{E}\left\{\left\langle G_{t} Y(t), Y(t)\right\rangle+\int_{t}^{T}[\langle Q(s) Y(s), Y(s)\rangle+\langle N(s) Z(s), Z(s)\rangle\right. \\
& +\langle R(s) u(s), u(s)\rangle] \mathrm{d} s\}
\end{aligned}
$$

where $G_{t}: \Omega \rightarrow \mathbb{S}^{n}$ is a bounded $\mathcal{F}_{t}$-measurable random variable, $Q, N \in L_{\mathbb{F}}^{\infty}\left(0, T ; \mathbb{S}_{+}^{n}\right), R \in L_{\mathbb{F}}^{\infty}\left(0, T ; \mathbb{S}_{+}^{m}\right)$, and $\langle\cdot, \cdot\rangle$ denotes the Frobenius inner product of two matrices. With the state equation (1.1) and the cost functional (1.2), the backward linear-quadratic (LQ, for short) optimal control problem can be stated as follows.

Problem (BLQ). For given terminal state $\xi \in L_{\mathcal{F}_{T}}^{2}\left(\Omega ; \mathbb{R}^{n}\right)$, find a control $u^{*} \in \mathcal{U}[t, T]$ such that

$$
J\left(t, \xi ; u^{*}\right)=\inf _{u \in \mathcal{U}[t, T]} J(t, \xi ; u) \equiv V(t, \xi) .
$$

A control $u^{*} \in \mathcal{U}[t, T]$ satisfying (1.3) is called an optimal control of Problem (BLQ) for the terminal state $\xi$; the corresponding state process $\left(Y^{*}, Z^{*}\right) \equiv\left(Y^{\xi, u^{*}}, Z^{\xi, u^{*}}\right)$ is called an optimal state process; the three-tuple $\left(Y^{*}, Z^{*}, u^{*}\right)$ is called an optimal triple; and the function $V$ is called the value function of Problem (BLQ).

The (forward) stochastic LQ problem (SLQ problem, for short) is a classical and fundamental problem in control theory, which was initially studied by Wonham [23] in 1968. In the book ([25], Chap. 6), it shows that under the standard conditions, the SLQ problem with deterministic coefficients is (uniquely) solvable; the optimality system, which is a coupled forward-backward stochastic differential equation, can be decoupled; and the optimal control can be further represented as a linear feedback of the current state, in terms of the solution to the associated Riccati equation. Another feature of SLQ problems is that the positive definiteness assumption on the weighting matrix of the control is not necessary for its solvability. This type of SLQ problems is called indefinite SLQ problems, see Chen-Li-Zhou [4] and some follow-up works [1, 6, 16, 21]. When the coefficients 
and weighting matrices are allowed to be random, the associated stochastic Riccati equation becomes a fully nonlinear BSDE with quadratic growth (see [3]), whose solvability was collected by Peng [14] in his list of open problems on BSDEs. Since then many efforts have been devoted to the SLQ problem with random coefficients; see Chen-Yong [5], Kohlmann-Tang [9], Tang [19, 20], Sun-Xiong-Yong [17], for instance. It is particularly worthy to mention that in [19], Tang first obtained a satisfactory solvability result for this type of stochastic Riccati equation under the standard conditions, which serves as a foundation for our study on Problem (BLQ). For more detailed history of SLQ problems, we refer the reader to the books by Yong-Zhou [25] and Sun-Yong [18].

The LQ optimal control problem for BSDEs with deterministic coefficients and weighting matrices was initially studied by Lim-Zhou [12] in 2001. The theory of backward LQ optimal control problem has important applications in mathematical finance, especially in financial investment problems with future conditions (as random variables) specified; see, $[11,12,15,25]$. It also has a great potential in studying stochastic differential games, as a backward LQ optimal control problem arises naturally when we consider the game in a leaderfollower manner; see for example, [24]. Because of these facts, there has been extensive research on the LQ optimal control (and game) problems for BSDEs. See, for example, Huang-Wang-Wu [8], Wang-Xiao-Xiong [22], Du-Huang-Wu [7], Li-Sun-Xiong [10], and Bi-Sun-Xiong [2]. Note that in our Problem (BLQ), the coefficients of (1.1) and the weighting matrices in (1.2) are allowed to be random. This feature makes it more complicated and difficult to study. Since it was proposed by Lim-Zhou in [12], there are few significative results on Problem (BLQ) with random coefficients so far.

One difficulty in solving random-coefficient backward LQ Problems is the solvability of the stochastic Riccati equation:

$$
\left\{\begin{aligned}
\mathrm{d} \Sigma(s)= & {\left[\Sigma A^{\top}+A \Sigma+\Sigma Q \Sigma-B R^{-1} B^{\top}+\Lambda N\left(I_{n}+\Sigma N\right)^{-1} \Lambda-C\left(I_{n}+\Sigma N\right)^{-1} \Sigma C^{\top}\right.} \\
& \left.-C\left(I_{n}+\Sigma N\right)^{-1} \Lambda-\Lambda\left(I_{n}+N \Sigma\right)^{-1} C^{\top}\right] \mathrm{d} s-\Lambda \mathrm{d} W(s), \quad s \in[0, T], \\
\Sigma(T)= & 0,
\end{aligned}\right.
$$

where the argumet $s$ is suppressed for notational simplicity (and we will frequently do so in the sequel if no confusion occurs). Different from the deterninistic case studied in [12], equation (1.4) is now a fully nonlinear BSDE with quadratic growth in $\Lambda$. Due to the presence of $\Lambda$ and $\left(I_{n}+N \Sigma\right)^{-1}$, the perturbed method used in [12] cannot be easily applied to our Problem (BLQ). To be more convincing, let us elaborate the difficulty we encountered in detail. It is very hard to directly establish the solvability of (1.4). However, thanks to the results on solvability of the stochastic Riccati equation for forward LQ optimal control problems (see [17, 19]), the following perturbed equation is easily seen to have a unique adapted solution $\left(\Sigma_{\varepsilon}, \Lambda_{\varepsilon}\right)$ for each $\varepsilon>0$ (see (5.42)):

$$
\left\{\begin{aligned}
\mathrm{d} \Sigma_{\varepsilon}(s)= & {\left[\Sigma_{\varepsilon} A^{\top}+A \Sigma_{\varepsilon}+\Sigma_{\varepsilon} Q \Sigma_{\varepsilon}-B R^{-1} B^{\top}+\Lambda_{\varepsilon} N\left(I_{n}+\Sigma_{\varepsilon} N\right)^{-1} \Lambda_{\varepsilon}-C\left(I_{n}+\Sigma_{\varepsilon} N\right)^{-1} \Sigma_{\varepsilon} C^{\top}\right.} \\
& \left.-C\left(I_{n}+\Sigma_{\varepsilon} N\right)^{-1} \Lambda_{\varepsilon}-\Lambda_{\varepsilon}\left(I_{n}+N \Sigma_{\varepsilon}\right)^{-1} C^{\top}\right] \mathrm{d} s-\Lambda_{\varepsilon} \mathrm{d} W(s) \\
\Sigma_{\varepsilon}(T)= & \varepsilon I_{n}
\end{aligned}\right.
$$

The first componet $\Sigma_{\varepsilon}$ of the solution is bounded, positive semi-definite, and monotone in $\varepsilon$. So the limit $\Sigma(s) \triangleq \lim _{\varepsilon \rightarrow 0} \Sigma_{\varepsilon}(s)$ exits for a.e. $s \in[0, T]$, a.s. If all the coefficients are deterministic, then $\Lambda_{\varepsilon}$ is identically zero for every $\varepsilon>0$ and hence $(\Sigma, 0)$ is a solution of (1.4). But in the random-coefficient case, the $\Lambda_{\varepsilon}$ 's are nonzero stochastic processes, even not bounded. The standard stability estimate also fails here since (1.5) is a multi-dimensional quadratic BSDE. The convergence of $\Lambda_{\varepsilon}$ therefore becomes very unclear. Instead of stubbornly proving the convergence of $\Lambda_{\varepsilon}$, we shall combine the above perturbed approach with a new method of undetermined coefficients to obtain the existence of a $\Lambda$, which, together with the limit of $\Sigma_{\varepsilon}$, gives the 
adapted solution of (1.4). We point out that although our work benefits from the results of Lim-Zhou [12] and Tang [19] a lot, it is by no means a simple extension of the backward LQ problem from deterministic case to the random one.

On the other hand, for deterministic-coefficient backward LQ Problems, it has been shown in $[10,12]$ that, in order to construct the optimal control, besides an ordinary Riccati equation, one need also consider an associated uncontrolled BSDE with bounded deterministic coefficients. Another difficulty is that for our Problem (BLQ), we have to consider the solvability of a BSDE with unbounded random coefficients. To our best knowledge, there are only a few papers dealing with such kind of BSDEs, and no existing results ensure the existence of an adapted solution to the BSDE associated with Problem (BLQ).

The purpose of this paper is to overcome the above difficulties and to give a complete solution of Problem (BLQ) under the following condition: For some $\delta>0$,

$$
Q(s) \geqslant 0, \quad N(s) \geqslant \delta I_{n}, \quad R(s) \geqslant \delta I_{m}, \quad s \in[0, T] .
$$

We shall show that Problem (BLQ) is uniquely solvable and establish the global solvability of the stochastic Riccati equation (1.4) under the condition (1.6). With the adapted solution to (1.4), we further introduce a decoupled system of forward-backward stochastic differential equations (FBSDEs, for short) with unbounded random coefficients and establish its unique solvability. Then we provide an explicit representation for the unique optimal control of Problem (BLQ), in terms of the solutions to (1.4) and the decoupled system of FBSDEs.

The rest of this paper is organized as follows. In Section 2, we collect some preliminary results of BSDEs. Section 3 is devoted to deriving the optimality system for Problem (BLQ) and establishing its unique solvability. To decouple the optimality system, we introduce a new stochastic Riccati-type equation and a decoupled system of FBSDEs with unbounded coefficients in Section 4. Finally, we establish the solvabilities of the stochastic Riccati equation and the decoupled system of FBSDEs in Section 5.

\section{Preliminaries}

Throughout this paper, $\mathbb{R}^{n \times m}$ is the Euclidean space consisting of $n \times m$ real matrices, endowed with the Frobenius inner product $\langle M, N\rangle \triangleq \operatorname{tr}\left[M^{\top} N\right]$, where $M^{\top}$ and $\operatorname{tr}(M)$ stand for the transpose and the trace of $M$, respectively. The identity matrix of size $n$ is denoted by $I_{n}$. When $m=1$, we simply write $\mathbb{R}^{n \times 1}$ as $\mathbb{R}^{n}$. If there is no confusion, we shall use $\langle\cdot, \cdot\rangle$ for inner products in possibly different Hilbert spaces and denote by $|\cdot|$ the norm induced by $\langle\cdot, \cdot\rangle$. Besides the notation introduced in Section 1, the following notation will be also frequently used in this paper:

$$
\begin{aligned}
L_{\mathcal{F}_{t}}^{\infty}(\Omega ; \mathbb{H}) & =\left\{\xi: \Omega \rightarrow \mathbb{H} \mid \xi \in \mathcal{F}_{t} \text { is bounded }\right\} ; \\
L_{\mathbb{F}}^{\infty}(\Omega ; C([t, T] ; \mathbb{H})) & =\{\varphi:[t, T] \times \Omega \rightarrow \mathbb{H} \mid \varphi \in \mathbb{F} \text { is continuous and bounded }\} ; \\
L_{\mathbb{F}}^{1}\left(\Omega ; L^{2}(t, T ; \mathbb{H})\right) & =\left\{\varphi:[t, T] \times \Omega \rightarrow \mathbb{H} \mid \varphi \in \mathbb{F} \text { and } \mathbb{E}\left[\int_{t}^{T}|\varphi(s)|^{2} \mathrm{~d} s\right]^{\frac{1}{2}}<\infty\right\} ; \\
L_{\mathbb{F}}^{2}(t, T ; \mathbb{H}) & =\left\{\varphi:[t, T] \times \Omega \rightarrow \mathbb{H} \mid \varphi \in \mathbb{F} \text { and } \mathbb{E} \int_{t}^{T}|\varphi(s)|^{2} \mathrm{~d} s<\infty\right\} ; \\
L_{\mathbb{F}}^{2}(\Omega ; C([t, T] ; \mathbb{H})) & =\left\{\varphi:[t, T] \times \Omega \rightarrow \mathbb{H} \mid \varphi \in \mathbb{F} \text { is continuous and } \mathbb{E}\left[\sup _{t \leqslant s \leqslant T}|\varphi(s)|^{2}\right]<\infty\right\} .
\end{aligned}
$$

For $M, N \in \mathbb{S}^{n}$, we use the notation $M \geqslant N$ (respectively, $M>N$ ) to indicate that $M-N$ is positive semidefinite (respectively, positive definite). Further, for an $\mathbb{S}^{n}$-valued measurable function $F$ on $[t, T]$, we write

$$
\begin{array}{lll}
F \geqslant 0 & \text { if } F(s) \geqslant 0, & \text { a.e. } s \in[t, T], \\
F>0 & \text { if } F(s)>0, & \text { a.e. } s \in[t, T], \\
F \gg 0 & \text { if } F(s) \geqslant \delta I_{n}, & \text { a.e. } s \in[t, T], \text { for some } \delta>0 .
\end{array}
$$


We will say that $F$ is uniformly positive definite if $F \gg 0$.

For the state system (1.1) and the cost functional (1.2), we impose the following assumptions.

(H1). The coefficients of the state equation (1.1) satisfy

$$
A, C \in L_{\mathbb{F}}^{\infty}\left(0, T ; \mathbb{R}^{n \times n}\right), \quad B \in L_{\mathbb{F}}^{\infty}\left(0, T ; \mathbb{R}^{n \times m}\right) .
$$

(H2). The weighting coefficients in the cost functional (1.2) satisfy

$$
Q, N \in L_{\mathbb{F}}^{\infty}\left(0, T ; \mathbb{S}_{+}^{n}\right), \quad R \in L_{\mathbb{F}}^{\infty}\left(0, T ; \mathbb{S}_{+}^{m}\right), \quad G_{t} \in L_{\mathcal{F}_{t}}^{\infty}\left(\Omega ; \mathbb{S}_{+}^{n}\right)
$$

Moreover, there exists a constant $\delta>0$ such that

$$
R(s) \geqslant \delta I_{m}, \quad \text { a.e. } s \in[0, T] \text {, a.s. }
$$

We now present a result concerning the well-posedness of the state equation (1.1).

Theorem 2.1. Let (H1) hold. Then for any terminal state $\xi \in L_{\mathcal{F}_{T}}^{2}\left(\Omega ; \mathbb{R}^{n}\right)$ and control $u \in \mathcal{U}[t, T]$, state equation (1.1) admits a unique adapted solution

$$
(Y, Z) \equiv\left(Y^{\xi, u}, Z^{\xi, u}\right) \in L_{\mathbb{F}}^{2}\left(\Omega ; C\left([t, T] ; \mathbb{R}^{n}\right)\right) \times L_{\mathbb{F}}^{2}\left(t, T ; \mathbb{R}^{n}\right) .
$$

Moreover, there exists a constant $K>0$, independent of $t, \xi$ and $u$, such that

$$
\begin{aligned}
& \mathbb{E}\left[\sup _{t \leqslant s \leqslant T}|Y(s)|^{2}+\int_{t}^{T}|Z(s)|^{2} \mathrm{~d} s\right] \leqslant K \mathbb{E}\left[|\xi|^{2}+\int_{t}^{T}|u(s)|^{2} \mathrm{~d} s\right], \\
& \sup _{t \leqslant s \leqslant T} \mathbb{E}_{t}|Y(s)|^{2}+\mathbb{E}_{t} \int_{t}^{T}|Z(s)|^{2} \mathrm{~d} s \leqslant K \mathbb{E}_{t}\left[|\xi|^{2}+\int_{t}^{T}|u(s)|^{2} \mathrm{~d} s\right], \quad \text { a.s., }
\end{aligned}
$$

where $\mathbb{E}_{t}[\cdot]=\mathbb{E}\left[\cdot \mid \mathcal{F}_{t}\right]$ is the conditional expectation operator.

Proof. It is standard to obtain the existence and uniqueness of the adapted solution to (1.1) and the estimate (2.1). The details and proofs of this reslut can be found in [25]. We only sketch the proof of estimate (2.2) here. Applying Itô's formula to $s \mapsto|Y(s)|^{2}$ yields that

$$
\begin{aligned}
& |Y(s)|^{2}+\int_{s}^{T}|Z(r)|^{2} \mathrm{~d} r+2 \int_{s}^{T} Y(r)^{\top} Z(r) \mathrm{d} W(r) \\
& \quad=|\xi|^{2}+2 \int_{s}^{T} Y(r)^{\top}[A(r) Y(r)+B(r) u(r)+C(r) Z(r)] \mathrm{d} r, \quad s \in[t, T] .
\end{aligned}
$$

Taking conditional expectations with respect to $\mathcal{F}_{t}$ on the both sides of $(2.3)$ and by $(\mathrm{H} 1)$, we get

$$
\begin{aligned}
& \mathbb{E}_{t}|Y(s)|^{2}+\mathbb{E}_{t} \int_{s}^{T}|Z(r)|^{2} \mathrm{~d} r \\
& \quad \leqslant K \mathbb{E}_{t}|\xi|^{2}+K \mathbb{E}_{t}\left\{\int_{s}^{T}\left[|Y(r)|^{2}+|Y(r)||Z(r)|+|Y(r)||u(r)|\right] \mathrm{d} r\right\}, \quad s \in[t, T] .
\end{aligned}
$$


By Young inequality, it is clearly seen from (2.4) that

$$
\begin{aligned}
& \mathbb{E}_{t}|Y(s)|^{2}+\mathbb{E}_{t} \int_{s}^{T}|Z(r)|^{2} \mathrm{~d} r \\
& \quad \leqslant K \mathbb{E}_{t}|\xi|^{2}+K \mathbb{E}_{t}\left\{\int_{s}^{T}\left[|Y(r)|^{2}+|u(r)|^{2}\right] \mathrm{d} r\right\}+\frac{1}{2} \mathbb{E}_{t} \int_{s}^{T}|Z(r)|^{2} \mathrm{~d} r, \quad s \in[t, T],
\end{aligned}
$$

which implies that

$$
\mathbb{E}_{t}|Y(s)|^{2}+\mathbb{E}_{t} \int_{s}^{T}|Z(r)|^{2} \mathrm{~d} r \leqslant K \mathbb{E}_{t}|\xi|^{2}+K \mathbb{E}_{t}\left\{\int_{s}^{T}\left[|Y(r)|^{2}+|u(r)|^{2}\right] \mathrm{d} r\right\}, \quad s \in[t, T] .
$$

The estimate (2.2) then follows from Grönwall's inequality immediately.

Under (H1), Theorem 2.1 shows that for any $\xi \in L_{\mathcal{F}_{T}}^{2}\left(\Omega ; \mathbb{R}^{n}\right)$ and $u \in \mathcal{U}[t, T]$, state equation (1.1) admits a unique adapted solution $(Y, Z) \in L_{\mathbb{F}}^{2}\left(\Omega ; C\left([t, T] ; \mathbb{R}^{n}\right)\right) \times L_{\mathbb{F}}^{2}\left(t, T ; \mathbb{R}^{n}\right)$. If, in addition, (H2) holds, then the random variables on the right-hand side of (1.2) are integrable and hence Problem (BLQ) is well-posed. When the coefficients and the weighting matrices reduce to deterministic functions, (H1)-(H2) are same as the Assumption (A1) in Lim-Zhou [12]. Moreover, (H2) implies the mapping $u \mapsto J(t, \xi ; u)$ is uniformly convex, which plays an important role in establishing the unique solvability of Problem (BLQ).

\section{Optimality systems And COUPLED FBSDEs}

In this section, we shall derive the optimality system for the optimal control of Problem (BLQ) by a variational method and then study the unique solvability of the optimality system from a Hilbert space point of view.

Theorem 3.1. Suppose that (H1) and (H2) hold. Then for any given terminal state $\xi \in L_{\mathcal{F}_{T}}^{2}\left(\Omega ; \mathbb{R}^{n}\right), u^{*} \in \mathcal{U}[t, T]$ is optimal for Problem (BLQ) if and only if the adapted solution $\left(Y^{*}, Z^{*}, X^{*}\right)$ to the following FBSDE

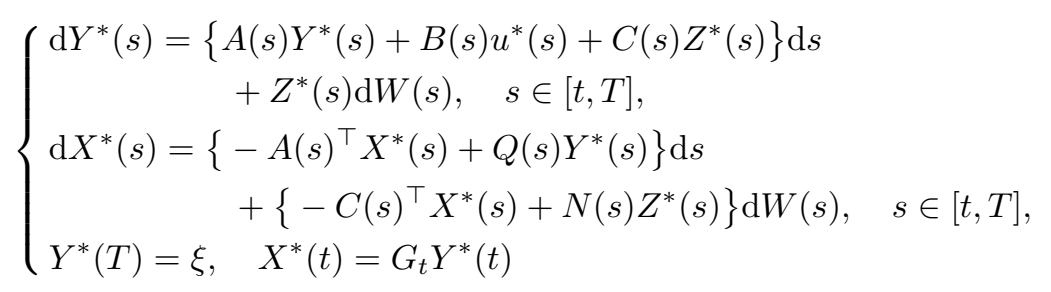

satisfies the following stationary condition:

$$
R(s) u^{*}(s)-B(s)^{\top} X^{*}(s)=0, \quad \text { a.e. } s \in[t, T] \text {, a.s. }
$$

Proof. By the definition of Problem (BLQ), $u^{*}$ is an optimal control if and only if

$$
J\left(t, \xi ; u^{*}\right) \leqslant J\left(t, \xi ; u^{*}+\varepsilon u\right), \quad \forall \varepsilon \in \mathbb{R}, u \in \mathcal{U}[t, T] .
$$

For any fixed but arbitrary $\varepsilon \in \mathbb{R}$ and $u \in \mathcal{U}[t, T]$, let $\left(Y_{\varepsilon}, Z_{\varepsilon}\right)$ be the adapted solution of BSDE (1.1) corresponding to the terminal state $\xi$ and control $u^{*}+\varepsilon u$; that is

$$
\left\{\begin{aligned}
\mathrm{d} Y_{\varepsilon}(s)= & \left\{A(s) Y_{\varepsilon}(s)+B(s)\left(u^{*}(s)+\varepsilon u(s)\right)+C(s) Z_{\varepsilon}(s)\right\} \mathrm{d} s \\
& +Z_{\varepsilon}(s) \mathrm{d} W(s), \quad s \in[t, T] \\
Y_{\varepsilon}(T)= & \xi
\end{aligned}\right.
$$


Let $(Y, Z)$ be the adapted solution of the following BSDE:

$$
\left\{\begin{aligned}
\mathrm{d} Y(s)= & \{A(s) Y(s)+B(s) u(s)+C(s) Z(s)\} \mathrm{d} s \\
& +Z(s) \mathrm{d} W(s), \quad s \in[t, T], \\
Y(T)= & 0 .
\end{aligned}\right.
$$

By the linearity of BSDEs (3.4), (3.5), (3.1) and the uniqueness of the adapted solution to BSDE (3.4), we get

$$
Y_{\varepsilon}=Y^{*}+\varepsilon Y, \quad Z_{\varepsilon}=Z^{*}+\varepsilon Z .
$$

Then it is straightforward to deduce the following representation of the difference $J\left(t, \xi ; u^{*}+\varepsilon u\right)-J\left(t, \xi ; u^{*}\right)$ :

$$
\begin{aligned}
J\left(t, \xi ; u^{*}+\varepsilon u\right)-J\left(t, \xi ; u^{*}\right) \\
=\varepsilon^{2} \mathbb{E}\left\{\left\langle G_{t} Y(t), Y(t)\right\rangle+\int_{t}^{T}(\langle Q Y, Y\rangle+\langle N Z, Z\rangle+\langle R u, u\rangle) \mathrm{d} s\right\} \\
\quad+2 \varepsilon \mathbb{E}\left\{\left\langle G_{t} Y^{*}(t), Y(t)\right\rangle+\int_{t}^{T}\left(\left\langle Q Y^{*}, Y\right\rangle+\left\langle N Z^{*}, Z\right\rangle+\left\langle R u^{*}, u\right\rangle\right) \mathrm{d} s\right\} .
\end{aligned}
$$

Thus the condition (3.3) is equivalent to

$$
\begin{gathered}
\varepsilon^{2} \mathbb{E}\left\{\left\langle G_{t} Y(t), Y(t)\right\rangle+\int_{t}^{T}(\langle Q Y, Y\rangle+\langle N Z, Z\rangle+\langle R u, u\rangle) \mathrm{d} s\right\}+2 \varepsilon \mathbb{E}\left\{\left\langle G_{t} Y^{*}(t), Y(t)\right\rangle\right. \\
\left.+\int_{t}^{T}\left(\left\langle Q Y^{*}, Y\right\rangle+\left\langle N Z^{*}, Z\right\rangle+\left\langle R u^{*}, u\right\rangle\right) \mathrm{d} s\right\} \geqslant 0, \quad \forall \varepsilon \in \mathbb{R}, u \in \mathcal{U}[t, T] .
\end{gathered}
$$

It is clearly seen from $(\mathrm{H} 2)$ that

$$
\mathbb{E}\left\{\left\langle G_{t} Y(t), Y(t)\right\rangle+\int_{t}^{T}(\langle Q Y, Y\rangle+\langle N Z, Z\rangle+\langle R u, u\rangle) \mathrm{d} s\right\} \geqslant 0, \quad \forall u \in \mathcal{U}[t, T]
$$

Note that for any fixed $u$ and $\xi$, the left-hand term of (3.8) could be regarded as a quadratic polynomial of the variable $\varepsilon$. Hence, (3.8) holds if and only if

$$
\mathbb{E}\left\{\left\langle G_{t} Y^{*}(t), Y(t)\right\rangle+\int_{t}^{T}\left(\left\langle Q Y^{*}, Y\right\rangle+\left\langle N Z^{*}, Z\right\rangle+\left\langle R u^{*}, u\right\rangle\right) \mathrm{d} s\right\}=0, \quad \forall u \in \mathcal{U}[t, T]
$$

By applying Itô's formula to $s \mapsto\left\langle X^{*}(s), Y(s)\right\rangle$ on $[t, T]$ and then taking expectation, we get

$$
\mathbb{E}\left\langle G_{t} Y^{*}(t), Y(t)\right\rangle=\mathbb{E}\left\langle X^{*}(t), Y(t)\right\rangle=-\mathbb{E} \int_{t}^{T}\left(\left\langle Q Y^{*}, Y\right\rangle+\left\langle B^{\top} X^{*}, u\right\rangle+\left\langle N Z^{*}, Z\right\rangle\right) \mathrm{d} s .
$$

Substituting the above into (3.9) yields that

$$
\mathbb{E} \int_{t}^{T}\left\langle R u^{*}-B^{\top} X^{*}, u\right\rangle d s=0, \quad \forall u \in \mathcal{U}[t, T]
$$

which implies that the stationary condition (3.2) holds. By reversing the above arguments, the sufficiency of (3.2) follows easily. 
The system (3.1), together with the stationary condition (3.2), is referred to as the optimality system for Problem (BLQ). For any given $u^{*} \in \mathcal{U}[t, T]$, the system (3.1) is a decoupled FBSDE. However, note that the optimal control $u^{*}$ necessarily satisfies the stationary condition (3.2), which is equivalent to

$$
u^{*}(s)=R(s)^{-1} B(s)^{\top} X^{*}(s), \quad \text { a.e. } s \in[t, T], \text { a.s. }
$$

Substituting the above into (3.1), the optimality system becomes a coupled FBSDE as follows:

$$
\left\{\begin{aligned}
& \mathrm{d} Y^{*}(s)=\{\left.A(s) Y^{*}(s)+B(s) R(s)^{-1} B(s)^{\top} X^{*}(s)+C(s) Z^{*}(s)\right\} \mathrm{d} s \\
&+Z^{*}(s) \mathrm{d} W(s), \quad s \in[t, T] \\
& \mathrm{d} X^{*}(s)=\{\left.-A(s)^{\top} X^{*}(s)+Q(s) Y^{*}(s)\right\} \mathrm{d} s \\
&+\left\{-C(s)^{\top} X^{*}(s)+N(s) Z^{*}(s)\right\} \mathrm{d} W(s), \quad s \in[t, T], \\
& Y^{*}(T)=\xi, \quad X^{*}(t)=G_{t} Y^{*}(t) .
\end{aligned}\right.
$$

In the subsequent analysis, we shall consider the well-posedness of FBSDE (3.12). To begin with, we present a unique solvability result of Problem (BLQ).

Theorem 3.2. Suppose that (H1) and $(\mathrm{H} 2)$ hold. Then for any terminal state $\xi \in L_{\mathcal{F}_{T}}^{2}\left(\Omega ; \mathbb{R}^{n}\right)$, Problem (BLQ) admits a unique optimal control.

Proof. For any $u \in \mathcal{U}[t, T]$, consider the following BSDE:

$$
\left\{\begin{aligned}
\mathrm{d} Y^{0, u}(s)= & \left\{A(s) Y^{0, u}(s)+B(s) u(s)+C(s) Z^{0, u}(s)\right\} \mathrm{d} s \\
& +Z^{0, u}(s) \mathrm{d} W(s), \quad s \in[t, T] \\
Y^{0, u}(T)= & 0
\end{aligned}\right.
$$

By Theorem 2.1, the above BSDE admits a unique adapted solution $\left(Y^{0, u}, Z^{0, u}\right) \in L_{\mathbb{F}}^{2}\left(\Omega ; C\left([t, T] ; \mathbb{R}^{n}\right)\right) \times$ $L_{\mathbb{F}}^{2}\left(t, T ; \mathbb{R}^{n}\right)$. By the linearity of $\operatorname{BSDE}(3.13)$, we can define two bounded linear operators $\mathcal{L}: \mathcal{U}[t, T] \rightarrow$ $L_{\mathbb{F}}^{2}\left(\Omega ; C\left([t, T] ; \mathbb{R}^{n}\right)\right) \times L_{\mathbb{F}}^{2}\left(t, T ; \mathbb{R}^{n}\right)$ and $\mathcal{M}: \mathcal{U}[t, T] \rightarrow L_{\mathcal{F}_{t}}^{2}\left(\Omega ; \mathbb{R}^{n}\right)$ as follows:

$$
\mathcal{L} u=\left(Y^{0, u}, Z^{0, u}\right), \quad \mathcal{M} u=Y^{0, u}(t), \quad u \in \mathcal{U}[t, T] .
$$

Also we can define the linear operators $\mathcal{N}: L_{\mathcal{F}_{T}}^{2}\left(\Omega ; \mathbb{R}^{n}\right) \rightarrow L_{\mathbb{F}}^{2}\left(\Omega ; C\left([t, T] ; \mathbb{R}^{n}\right)\right) \times L_{\mathbb{F}}^{2}\left(t, T ; \mathbb{R}^{n}\right)$ and $\mathcal{O}:$ $L_{\mathcal{F}_{T}}^{2}\left(\Omega ; \mathbb{R}^{n}\right) \rightarrow L_{\mathcal{F}_{t}}^{2}\left(\Omega ; \mathbb{R}^{n}\right)$ as follows:

$$
\mathcal{N} \xi=\left(Y^{\xi, 0}, Z^{\xi, 0}\right), \quad \mathcal{O} \xi=Y^{\xi, 0}(t), \quad \xi \in L_{\mathcal{F}_{T}}^{2}\left(\Omega ; \mathbb{R}^{n}\right)
$$

with $\left(Y^{\xi, 0}, Z^{\xi, 0}\right)$ being the adapted solution of the following BSDE:

$$
\left\{\begin{aligned}
\mathrm{d} Y^{\xi, 0}(s) & =\left\{A(s) Y^{\xi, 0}(s)+C(s) Z^{\xi, 0}(s)\right\} \mathrm{d} s+Z^{\xi, 0}(s) \mathrm{d} W(s), \quad s \in[t, T], \\
Y^{\xi, 0}(T) & =\xi
\end{aligned}\right.
$$

Observe that for any $(\xi, u) \in L_{\mathcal{F}_{T}}^{2}\left(\Omega ; \mathbb{R}^{n}\right) \times \mathcal{U}[t, T]$, the sum $\left(Y^{0, u}+Y^{\xi, 0}, Z^{0, u}+Z^{\xi, 0}\right)$ satisfies BSDE (1.1). By the uniqueness of the adapted solution to $\operatorname{BSDE}(1.1)$, we get

$$
(Y, Z)=\left(Y^{0, u}+Y^{\xi, 0}, Z^{0, u}+Z^{\xi, 0}\right)=\mathcal{L} u+\mathcal{N} \xi
$$


In particular, the initial value $Y(t)$ is given by

$$
Y(t)=Y^{0, u}(t)+Y^{\xi, 0}(t)=\mathcal{M} u+\mathcal{O} \xi
$$

Now let $\mathcal{A}^{*}$ denote the adjoint operator of a linear operator $\mathcal{A}$, and define the bounded linear operator $\mathcal{Q}$ : $L_{\mathbb{F}}^{2}\left(t, T ; \mathbb{R}^{n}\right) \times L_{\mathbb{F}}^{2}\left(t, T ; \mathbb{R}^{n}\right) \rightarrow L_{\mathbb{F}}^{2}\left(t, T ; \mathbb{R}^{n}\right) \times L_{\mathbb{F}}^{2}\left(t, T ; \mathbb{R}^{n}\right)$ by

$$
\mathcal{Q} \triangleq\left(\begin{array}{cc}
Q & 0 \\
0 & N
\end{array}\right)
$$

Then by the representations (3.17), (3.18), and (3.19), the cost functional (1.2) can be rewritten as follows:

$$
\begin{aligned}
J(t, \xi ; u)= & \mathbb{E}\left\{\left\langle G_{t} Y(t), Y(t)\right\rangle+\int_{t}^{T}\left[\left\langle\left(\begin{array}{cc}
Q(s) & 0 \\
0 & N(s)
\end{array}\right)\left(\begin{array}{l}
Y(s) \\
Z(s)
\end{array}\right),\left(\begin{array}{c}
Y(s) \\
Z(s)
\end{array}\right)\right\rangle\right.\right. \\
& +\langle R(s) u(s), u(s)\rangle] \mathrm{d} s\} \\
= & \left\langle G_{t}(\mathcal{M} u+\mathcal{O} \xi), \mathcal{M} u+\mathcal{O} \xi\right\rangle+\langle\mathcal{Q}(\mathcal{L} u+\mathcal{N} \xi), \mathcal{L} u+\mathcal{N} \xi\rangle+\langle R u, u\rangle \\
= & \left\langle\left(\mathcal{M}^{*} G_{t} \mathcal{M}+\mathcal{L}^{*} \mathcal{Q} \mathcal{L}+R\right) u, u\right\rangle+2\left\langle\left(\mathcal{O}^{*} G_{t} \mathcal{M}+\mathcal{N}^{*} \mathcal{Q L}\right) u, \xi\right\rangle \\
& +\left\langle\left(\mathcal{O}^{*} G_{t} \mathcal{O}+\mathcal{N}^{*} \mathcal{Q N}\right) \xi, \xi\right\rangle
\end{aligned}
$$

Since all the linear operators involved in the above are bounded, the map $u \mapsto J(t, \xi ; u)$ is continuous. Due to the facts that $\mathcal{Q} \geqslant 0, G_{t} \geqslant 0$ and $R \geqslant \delta I_{n}$ obtained from (H2), we have

$$
\left\langle\left(\mathcal{M}^{*} G_{t} \mathcal{M}+\mathcal{L}^{*} \mathcal{Q} \mathcal{L}+R\right) u, u\right\rangle \geqslant\langle R u, u\rangle=\mathbb{E} \int_{t}^{T}\langle R(s) u(s), u(s)\rangle d s \geqslant \delta \mathbb{E} \int_{t}^{T}|u(s)|^{2} \mathrm{~d} s,
$$

which implies the map $u \mapsto J(t, \xi ; u)$ is strictly convex, and that

$$
J(t, \xi ; u) \rightarrow \infty \quad \text { as } \quad \mathbb{E} \int_{t}^{T}|u(s)|^{2} \mathrm{~d} s \rightarrow \infty .
$$

Therefore, by the basic theorem in convex analysis, the unique solvability of Problem (BLQ), for any given terminal state $\xi \in L_{\mathcal{F}_{T}}^{2}\left(\Omega ; \mathbb{R}^{n}\right)$, is obtained.

Combining Theorem 3.1 with Theorem 3.2 together, we get the unique solvability of FBSDE (3.12) immediately.

Theorem 3.3. Suppose that (H1) and (H2) hold. Then for any terminal state $\xi \in L_{\mathcal{F}_{T}}^{2}\left(\Omega ; \mathbb{R}^{n}\right)$, the coupled FBSDE (3.12) admits a unique adapted solution $\left(Y^{*}, Z^{*}, X^{*}\right) \in L_{\mathbb{F}}^{2}\left(\Omega ; C\left([t, T] ; \mathbb{R}^{n}\right)\right) \times L_{\mathbb{F}}^{2}\left(t, T ; \mathbb{R}^{n}\right) \times$ $L_{\mathbb{F}}^{2}\left(\Omega ; C\left([t, T] ; \mathbb{R}^{n}\right)\right)$. Moreover, the unique optimal control of Problem (BLQ) for $\xi$ is given by

$$
u^{*}(s)=R(s)^{-1} B(s)^{\top} X^{*}(s), \quad s \in[t, T] .
$$

Remark 3.4. We emphasize that in $\operatorname{FBSDE}(3.12)$, the terminal state $Y^{*}(T)$ is an arbitrary $\mathcal{F}_{T}$-measurable random vector and the initial state $X^{*}(t)$ is determined by the initial value of $Y^{*}$, due to which FBSDE (3.12) is not Markovian even if its coefficients are deterministic functions. Thus the form of FBSDE (3.12) is a little different from the standard FBSDEs (see [13], for example). In particular, it will be interesting to give a "Four-Step Scheme" for this type of FBSDEs. 


\section{Decoupling, Riccati equation, BSDE and FSDE with UNBOUNDED COEFFICIENTS}

Since the optimality system is a fully coupled FBSDE, it usually becomes difficult to find the optimal control by solving (3.12) directly. Then, to construct an optimal control from the optimality system (3.12), a decoupling technique needs to be adopted. Thus, we now introduce the following stochastic Riccati-type equation:

$$
\left\{\begin{aligned}
\mathrm{d} \Sigma(s)= & {\left[\Sigma A^{\top}+A \Sigma+\Sigma Q \Sigma-B R^{-1} B^{\top}+\Lambda N\left(I_{n}+\Sigma N\right)^{-1} \Lambda-C\left(I_{n}+\Sigma N\right)^{-1} \Sigma C^{\top}\right.} \\
& \left.-C\left(I_{n}+\Sigma N\right)^{-1} \Lambda-\Lambda\left(I_{n}+N \Sigma\right)^{-1} C^{\top}\right] \mathrm{d} s-\Lambda \mathrm{d} W(s), \quad s \in[0, T], \\
\Sigma(T)= & 0 .
\end{aligned}\right.
$$

If the above equation is solvable with $(\Sigma, \Lambda)$ being a solution, we introduce the following BSDE:

$$
\left\{\begin{aligned}
\mathrm{d} \varphi(s)= & \left\{(A+\Sigma Q) \varphi-C\left(I_{n}+\Sigma N\right)^{-1} \beta+\Lambda N\left(I_{n}+\Sigma N\right)^{-1} \beta\right\} \mathrm{d} s \\
& -\beta \mathrm{d} W(s), \quad s \in[t, T], \\
\varphi(T)= & -\xi .
\end{aligned}\right.
$$

It is noteworthy that $\Lambda$ is merely square-integrable in general, thus (4.2) is a BSDE with unbounded coefficients. Suppose that BSDE (4.2) has a solution $(\varphi, \beta)$, we consider the following forward stochastic different equation (FSDE, for short):

$$
\left\{\begin{aligned}
\mathrm{d} X(s)=- & \left\{\left(A^{\top}+Q \Sigma\right) X+Q \varphi\right\} \mathrm{d} s+\left\{-C^{\top} X+N\left(I_{n}+\Sigma N\right)^{-1}\left(\Lambda+\Sigma C^{\top}\right) X\right. \\
& \left.+N\left(I_{n}+\Sigma N\right)^{-1} \beta\right\} \mathrm{d} W(s), \quad s \in[t, T], \\
X(t)=- & \left(I_{n}+G_{t} \Sigma(t)\right)^{-1} G_{t} \varphi(t) .
\end{aligned}\right.
$$

Similar to (4.2), the coefficients of FSDE (4.3) are also unbounded in general. Under the assumption that the above equations (4.1)-(4.2)-(4.3) are solvable, the following result provides a method of decoupling FBSDE (3.12). At first, we impose an additional assumption for the weighting matrix $N$ of cost functional (1.2).

(H3). There exist two constants $\delta, \lambda>0$ such that

$$
\delta I_{n} \leqslant N(s) \leqslant \lambda I_{n}, \quad \text { a.s., a.e. } s \in[0, T]
$$

or, equivalently,

$$
\frac{1}{\lambda} I_{n} \leqslant N(s)^{-1} \leqslant \frac{1}{\delta} I_{n}, \quad \text { a.s., a.e. } s \in[0, T]
$$

where $N(s)^{-1}$ stands for the inverse of $N(s)$.

Since $N$ is assumed to be bounded in (H2), the existence of $\lambda$ can follow from that easily. The non-degenerate assumption (i.e., $\delta I_{n} \leqslant N(s)$ ) is a technical condition (see (4.9)-(5.56) for some reasons). At the moment, we cannot improve it and we shall come back in our future publications.

Theorem 4.1. Let (H1)-(H2)-(H3) hold. Suppose that Riccati equation (4.1) has a solution $(\Sigma, \Lambda) \in$ $L_{\mathbb{F}}^{\infty}\left(\Omega ; C\left([0, T] ; \mathbb{S}_{+}^{n}\right)\right) \times L_{\mathbb{F}}^{2}\left(0, T ; \mathbb{S}^{n}\right)$ such that the corresponding decoupled system of BSDE (4.2) and FSDE (4.3) has a solution $(\varphi, \beta, X) \in L_{\mathbb{F}}^{2}\left(\Omega ; C\left([t, T] ; \mathbb{R}^{n}\right)\right) \times L_{\mathbb{F}}^{1}\left(\Omega ; L^{2}\left(t, T ; \mathbb{R}^{n}\right)\right) \times L_{\mathbb{F}}^{2}\left(\Omega ; C\left([t, T] ; \mathbb{R}^{n}\right)\right)$. Then the unique 
adapted solution $\left(Y^{*}, Z^{*}, X^{*}\right)$ of FBSDE (3.12) can be given by

$$
\left(Y^{*}, Z^{*}, X^{*}\right)=\left(-\Sigma X-\varphi,\left(I_{n}+\Sigma N\right)^{-1}\left(\Lambda X+\Sigma C^{\top} X+\beta\right), X\right),
$$

and the unique optimal control $u^{*}$ of Problems (BLQ) has the following explicit representation:

$$
u^{*}=R^{-1} B^{\top} X
$$

Proof. For convenience, we denote

$$
(\widehat{Y}, \widehat{Z}, \widehat{X}) \triangleq\left(-\Sigma X-\varphi,\left(I_{n}+\Sigma N\right)^{-1}\left(\Lambda X+\Sigma C^{\top} X+\beta\right), X\right) .
$$

Note that

$$
\int_{t}^{T}\left|\left(I_{n}+\Sigma N\right)^{-1}\left(\Lambda X+\Sigma C^{\top} X+\beta\right)\right|^{2} \mathrm{~d} s<\infty, \quad \text { a.s. }
$$

Define for each $k>1$ the stopping time (with the convention inf $\emptyset=\infty$ )

$$
\tau_{k}=\inf \left\{s \in[t, T] ; \int_{t}^{s}\left|\left(I_{n}+\Sigma N\right)^{-1}\left(\Lambda X+\Sigma C^{\top} X+\beta\right)\right|^{2} \mathrm{~d} r \geqslant k\right\} .
$$

By (H3), we have

$$
\begin{aligned}
& \mathbb{E} \int_{t}^{\tau_{k} \wedge T}\left|\left(I_{n}+\Sigma N\right)^{-1}\left(\Lambda X+\Sigma C^{\top} X+\beta\right)\right|^{2} \mathrm{~d} s \\
& \quad=\mathbb{E} \int_{t}^{\tau_{k} \wedge T}\left|N^{-1} N\left(I_{n}+\Sigma N\right)^{-1}\left(\Lambda X+\Sigma C^{\top} X+\beta\right)\right|^{2} \mathrm{~d} s \\
& \quad \leqslant K \mathbb{E} \int_{t}^{\tau_{k} \wedge T}\left|N\left(I_{n}+\Sigma N\right)\left(\Lambda X+\Sigma C^{\top} X+\beta\right)\right|^{2} \mathrm{~d} s .
\end{aligned}
$$

Recall that $X$ satisfies FSDE (4.3), we have by Itô's isometry that

$$
\begin{aligned}
& \mathbb{E} \int_{t}^{\tau_{k} \wedge T}\left|N\left(I_{n}+\Sigma N\right)\left(\Lambda X+\Sigma C^{\top} X+\beta\right)\right|^{2} \mathrm{~d} s \\
& \leqslant K \mathbb{E}\left|G_{t}\left(I_{n}+\Sigma(t) G_{t}\right)^{-1} \varphi(t)\right|^{2}+K \mathbb{E}\left|X\left(T \wedge \tau_{k}\right)\right|^{2} \\
&+K \mathbb{E} \int_{t}^{\tau_{k} \wedge T}\left[\left|\left(A^{\top}+Q \Sigma\right) X\right|^{2}+|Q \varphi|^{2}+\left|C^{\top} X\right|^{2}\right] \mathrm{d} s \\
& \leqslant K \mathbb{E}\left|G_{t}\left(I_{n}+\Sigma(t) G_{t}\right)^{-1} \varphi(t)\right|^{2}+K \mathbb{E}\left[\sup _{s \in[t, T]}|X(s)|^{2}\right] \\
&+K \mathbb{E} \int_{t}^{T}\left[\left|\left(A^{\top}+Q \Sigma\right) X\right|^{2}+|Q \varphi|^{2}+\left|C^{\top} X\right|^{2}\right] \mathrm{d} s \\
& \leqslant K \mathbb{E}\left[\sup _{s \in[t, T]}|X(s)|^{2}+\sup _{s \in[t, T]}|\varphi(s)|^{2}\right] .
\end{aligned}
$$


Combining the above with (4.9), by the definition of $\widehat{Z}$, we have

$$
\begin{aligned}
\mathbb{E} \int_{t}^{\tau_{k} \wedge T}|\widehat{Z}|^{2} \mathrm{~d} s & =\mathbb{E} \int_{t}^{\tau_{k} \wedge T}\left|\left(I_{n}+\Sigma N\right)^{-1}\left(\Lambda X+\Sigma C^{\top} X+\beta\right)\right|^{2} \mathrm{~d} s \\
& \leqslant K \mathbb{E}\left[\sup _{s \in[t, T]}|X(s)|^{2}+\sup _{s \in[t, T]}|\varphi(s)|^{2}\right]<\infty .
\end{aligned}
$$

Since $\lim _{k \rightarrow \infty} \tau_{k}=\infty$ almost surely and the right-hand side of the above inequality is independent of $\tau_{k}$, we conclude $\widehat{Z}=\left(I_{n}+\Sigma N\right)\left(\Lambda X+\Sigma C^{\top} X+\beta\right) \in L_{\mathbb{F}}^{2}\left(t, T ; \mathbb{R}^{n}\right)$ by letting $k \rightarrow \infty$. Then it is clear to see that $(\widehat{Y}, \widehat{Z}, \widehat{X}) \in L_{\mathbb{F}}^{2}\left(\Omega ; C\left([t, T] ; \mathbb{R}^{n}\right)\right) \times L_{\mathbb{F}}^{2}\left(t, T ; \mathbb{R}^{n}\right) \times L_{\mathbb{F}}^{2}\left(\Omega ; C\left([t, T] ; \mathbb{R}^{n}\right)\right)$.

By the uniqueness of the adapted solution to FBSDE (3.12) from Theorem 3.3, it suffices to verify that $(\widehat{Y}, \widehat{Z}, \widehat{X})$ satisfies FBSDE (3.12). By the definition (4.8) of $(\widehat{Y}, \widehat{Z}, \widehat{X})$ and equation (4.3) of $X, \widehat{X}$ satisfies

$$
\begin{aligned}
\mathrm{d} \widehat{X}(s)= & \left\{-A^{\top} \widehat{X}-Q[\Sigma \widehat{X}+\varphi]\right\} \mathrm{d} s \\
& +\left\{-C^{\top} \widehat{X}+N\left[\left(I_{n}+\Sigma N\right)^{-1}\left(\Lambda \widehat{X}+\Sigma C^{\top} \widehat{X}+\beta\right)\right]\right\} \mathrm{d} W(s) \\
= & \left\{-A^{\top} \widehat{X}+Q \hat{Y}\right\} \mathrm{d} s+\left\{-C^{\top} \widehat{X}+N \widehat{Z}\right\} \mathrm{d} W(s), \quad s \in[t, T] .
\end{aligned}
$$

Further, by the initial condition in (4.3), the initial value of $\widehat{X}$ is given by

$$
\begin{aligned}
\widehat{X}(t) & =X(t)=\left[I_{n}+G_{t} \Sigma(t)\right] X(t)-G_{t} \Sigma(t) X(t) \\
& =-\left[I_{n}+G_{t} \Sigma(t)\right]\left[I_{n}+G_{t} \Sigma(t)\right]^{-1} G_{t} \varphi(t)-G_{t} \Sigma(t) X(t) \\
& =G_{t}[-\varphi(t)-\Sigma(t) X(t)]=G_{t} \widehat{Y}(t) .
\end{aligned}
$$

Thus $\widehat{X}$ satisfies the same FSDE as $X^{*}$ with $\left(Y^{*}, Z^{*}\right)$ replaced by $(\widehat{Y}, \widehat{Z})$.

We now show that $(\widehat{Y}, \widehat{Z})$ satisfies the same BSDE as $\left(Y^{*}, Z^{*}\right)$ in $(3.12)$ with $X^{*}$ replaced by $\widehat{X}$. By applying Itô's formula to $s \mapsto \widehat{Y}(s) \equiv-\Sigma(s) X(s)-\varphi(s)$, some straightforward calculations yield that

$$
\begin{aligned}
\mathrm{d} \widehat{Y}(s)= & d[-\Sigma(s) X(s)-\varphi(s)] \\
= & \left\{-A(\Sigma X+\varphi)+B R^{-1} B^{\top} X+C\left(I_{n}+\Sigma N\right)^{-1}\left(\Lambda X+\Sigma C^{\top} X+\beta\right)\right. \\
& \left.+\Lambda N\left(I_{n}+\Sigma N\right)^{-1} \Sigma C^{\top} X-\Lambda C^{\top} X+\Lambda\left(I_{n}+N \Sigma\right)^{-1} C^{\top} X\right\} \mathrm{d} s \\
& +\left\{\Lambda X+\Sigma C^{\top} X+\beta-\Sigma N\left(I_{n}+\Sigma N\right)^{-1}\left(\Lambda X+\Sigma C^{\top} X+\beta\right)\right\} \mathrm{d} W(s) \\
\equiv & (I) d s+(I I) \mathrm{d} W(s) .
\end{aligned}
$$

Using the fact that

$$
\left(I_{n}+\Sigma N\right)^{-1} \Sigma=\Sigma\left(I_{n}+N \Sigma\right)^{-1}, \quad N \Sigma\left(I_{n}+N \Sigma\right)^{-1}-I_{n}=-\left(I_{n}+N \Sigma\right)^{-1},
$$

we have

$$
\begin{aligned}
\Lambda N & \left(I_{n}+\Sigma N\right)^{-1} \Sigma C^{\top} X-\Lambda C^{\top} X+\Lambda\left(I_{n}+N \Sigma\right)^{-1} C^{\top} X \\
& =\Lambda N \Sigma\left(I_{n}+N \Sigma\right)^{-1} C^{\top} X-\Lambda C^{\top} X+\Lambda\left(I_{n}+N \Sigma\right)^{-1} C^{\top} X \\
& =\Lambda\left[N \Sigma\left(I_{n}+N \Sigma\right)^{-1}-I_{n}\right] C^{\top} X+\Lambda\left(I_{n}+N \Sigma\right)^{-1} C^{\top} X \\
& =-\Lambda\left(I_{n}+N \Sigma\right)^{-1} C^{\top} X+\Lambda\left(I_{n}+N \Sigma\right)^{-1} C^{\top} X=0 .
\end{aligned}
$$


Then by the definition $(4.8)$ of $(\widehat{X}, \widehat{Y}, \widehat{Z})$, the drift term in (4.14) can be rewritten as

$$
\begin{aligned}
(I) & =-A(\Sigma X+\varphi)+B R^{-1} B^{\top} X+C\left(I_{n}+\Sigma N\right)^{-1}\left(\Lambda X+\Sigma C^{\top} X+\beta\right) \\
& =A \widehat{Y}+B R^{-1} B^{\top} \widehat{X}+C \widehat{Z}
\end{aligned}
$$

By the definition (4.8) of $\widehat{Z}$, the diffusion term in (4.14) can be expressed as

$$
\begin{aligned}
(I I) & =\left[I_{n}-\Sigma N\left(I_{n}+\Sigma N\right)^{-1}\right]\left(\Lambda X+\Sigma C^{\top} X+\beta\right) \\
& =\left(I_{n}+\Sigma N\right)^{-1}\left(\Lambda X+\Sigma C^{\top} X+\beta\right)=\widehat{Z} .
\end{aligned}
$$

Combining the above with (4.16), we can rewrite (4.14) as

$$
\mathrm{d} \widehat{Y}(s)=\left\{A \widehat{Y}+B R^{-1} B^{\top} \widehat{X}+C \widehat{Z}\right\} \mathrm{d} s+\widehat{Z} d W(s) .
$$

Moreover, by the terminal values of $\Sigma$ and $\varphi, \widehat{Y}$ satisfies the following terminal condition:

$$
\widehat{Y}(T)=-\Sigma(T) X(T)-\varphi(T)=\xi .
$$

It follows that $(\widehat{Y}, \widehat{Z})$ satisfies the same BSDE as $\left(Y^{*}, Z^{*}\right)$ with $X^{*}$ replaced by $\widehat{X}$. The proof is thus completed.

To illustrates the procedure for finding the optimal control by Theorem 4.1, we conclude this section by presenting the following simple example.

Example 4.2. Consider the one-dimensional controlled BSDE:

$$
\left\{\begin{aligned}
\mathrm{d} Y(s) & =\frac{1}{1+W(s)^{2}} u(s) d s+Z(s) \mathrm{d} W(s), \quad s \in[0, T] \\
Y(T) & =1
\end{aligned}\right.
$$

and the cost functional:

$$
J(0, \xi ; u)=\mathbb{E} \int_{0}^{T}\left[\frac{2+W(s)^{2}}{1+W(s)^{2}}|Z(s)|^{2}+\frac{2+W(s)^{2}}{1+W(s)^{2}}|u(s)|^{2}\right] \mathrm{d} s .
$$

Notice that

$$
0<\frac{1}{1+W(s)^{2}} \leqslant 1, \quad 1<\frac{2+W(s)^{2}}{1+W(s)^{2}} \leqslant 2, \quad s \in[0, T]
$$

Thus, the example satisfies the assumptions (H1)-(H2)-(H3). In the following, we are applying Theorem 4.1 to obtain the optimal control. The corresponding Riccati equation (4.1), BSDE (4.2), SDE (4.3) read:

$$
\left\{\begin{aligned}
\mathrm{d} \Sigma(s)= & {\left[-\frac{1}{\left[1+W(s)^{2}\right]\left[2+W(s)^{2}\right]}+\Lambda \frac{2+W(s)^{2}}{1+W(s)^{2}}\left(I_{n}+\Sigma \frac{2+W(s)^{2}}{1+W(s)^{2}}\right)^{-1} \Lambda\right] \mathrm{d} s } \\
& -\Lambda \mathrm{d} W(s), \quad s \in[0, T], \\
\Sigma(T)= & 0,
\end{aligned}\right.
$$




$$
\left\{\begin{aligned}
\mathrm{d} \varphi(s) & =\Lambda \frac{2+W(s)^{2}}{1+W(s)^{2}}\left(I_{n}+\Sigma \frac{2+W(s)^{2}}{1+W(s)^{2}}\right)^{-1} \beta d s-\beta \mathrm{d} W(s), \quad s \in[t, T] \\
\varphi(T) & =-1
\end{aligned}\right.
$$

and

$$
\left\{\begin{aligned}
d X(s)= & \left\{\frac{2+W(s)^{2}}{1+W(s)^{2}}\left(I_{n}+\Sigma \frac{2+W(s)^{2}}{1+W(s)^{2}}\right)^{-1} \Lambda X\right. \\
& \left.\quad+\frac{2+W(s)^{2}}{1+W(s)^{2}}\left(I_{n}+\Sigma \frac{2+W(s)^{2}}{1+W(s)^{2}}\right)^{-1} \beta\right\} \mathrm{d} W(s), \quad s \in[t, T], \\
X(t)= & 0 .
\end{aligned}\right.
$$

Note that $(-1,0,0)$ is the unique solution to the decoupled system of BSDE (4.20) and SDE (4.21). By the formula (4.7) in Theorem 4.1, the unique optimal control $u^{*}$ is given by

$$
u^{*}(s)=R(s)^{-1} B(s)^{\top} X(s) \equiv 0, \quad s \in[0, T]
$$

\section{Solvabilities of the Riccati equation, BSDE and FSDE with UNBOUNDED COEFFICIENTS}

If the solutions $(\Sigma, \Lambda, \varphi, \beta, X)$ of (4.1)-(4.2)-(4.3) are solved, then it immediately follows from Theorem 4.1 that FBSDE (3.12) can be decoupled and the unique optimal control of Problem (BLQ) can be represented explicitly. In this subsection, we shall establish the solvabilities of Riccati equation (4.1), BSDE (4.2), and FSDE (4.3).

We begin with two interesting results of the optimal control problems for forward SDEs, which will play a basic role in our subsequent analysis. For any given $\varepsilon>0$, consider the following stochastic Riccati equation:

$$
\left\{\begin{aligned}
d P_{\varepsilon}(s)=- & \left\{P_{\varepsilon} A+A^{T} P_{\varepsilon}+Q-\left[P_{\varepsilon}(B, C)+\left(0, \Pi_{\varepsilon}\right)\right]\right. \\
& \left.\times\left(\begin{array}{cc}
R & 0 \\
0 & N+P_{\varepsilon}
\end{array}\right)^{-1}\left[(B, C)^{\top} P_{\varepsilon}+\left(0, \Pi_{\varepsilon}\right)^{\top}\right]\right\}+\Pi_{\varepsilon} \mathrm{d} W(s), \quad s \in[0, T], \\
P_{\varepsilon}(T)=\varepsilon^{-1} I_{n} &
\end{aligned}\right.
$$

Note that (5.1) is the Riccati equation associated the following forward LQ problems: For the given $\varepsilon>0$, consider the following controlled FSDE:

$$
\left\{\begin{aligned}
\mathrm{d} X(s) & =\{A(s) X(s)+B(s) u(s)+C(s) v(s)\} \mathrm{d} s+v(s) \mathrm{d} W(s), \quad s \in[t, T], \\
X(t) & =\eta
\end{aligned}\right.
$$

and the cost functional:

$$
\begin{aligned}
J_{\varepsilon}(t, \eta ; u, v)=\mathbb{E}_{t}\{ & \int_{t}^{T}[\langle Q(s) X(s), X(s)\rangle+\langle N(s) v(s), v(s)\rangle+\langle R(s) u(s), u(s)\rangle] \mathrm{d} s \\
& \left.+\varepsilon^{-1}\langle X(T), X(T)\rangle\right\} .
\end{aligned}
$$


For any given $(t, \eta) \in[0, T] \times L_{\mathcal{F}_{t}}^{2}\left(\Omega ; \mathbb{R}^{n}\right)$, find a $\left(u^{*}, v^{*}\right) \in L_{\mathbb{F}}^{2}\left(t, T ; \mathbb{R}^{m}\right) \times L_{\mathbb{F}}^{2}\left(t, T ; \mathbb{R}^{n}\right)$ such that

$$
J_{\varepsilon}\left(t, \eta ; u^{*}, v^{*}\right)=\underset{u, v}{\operatorname{essinf}} J_{\varepsilon}(t, \eta ; u, v)=V_{\varepsilon}(t, \eta),
$$

as $(u, v)$ ranges over the space $L_{\mathbb{F}}^{2}\left(t, T ; \mathbb{R}^{m}\right) \times L_{\mathbb{F}}^{2}\left(t, T ; \mathbb{R}^{n}\right)$. Under $(\mathrm{H} 1)-(\mathrm{H} 2)-(\mathrm{H} 3)$, we have

$$
\left(\begin{array}{cc}
R & 0 \\
0 & N
\end{array}\right) \geqslant \delta I_{m+n}, \quad Q \geqslant 0, \quad \text { and } \quad \varepsilon^{-1} \geqslant 0
$$

Thus the above SLQ problems satisfies the so-called standard condition in the literature [17, 19, 25]. Then by [17], Theorem 6.2. (or [19], Thm. 5.3), Riccati equation (5.1) admits a unique solution $\left(P_{\varepsilon}, \Pi_{\varepsilon}\right) \in$ $L_{\mathbb{F}}^{\infty}\left(\Omega ; C\left([0, T] ; \mathbb{S}_{+}^{n}\right)\right) \times L_{\mathbb{F}}^{2}\left(0, T ; \mathbb{S}^{n}\right)$. The following result shows that $P_{\varepsilon}$ is uniformly positive definite (for the given $\varepsilon>0)$.

Proposition 5.1. Let (H1)-(H2)-(H3) hold. Then for any given $\varepsilon>0, P_{\varepsilon}$ is uniformly positive definite; that is

$$
P_{\varepsilon}(s) \geqslant \alpha_{\varepsilon} I_{n}, \quad \text { a.s., } s \in[0, T],
$$

for some $\alpha_{\varepsilon}>0$.

Proof. For the given $\varepsilon>0$, by (H2)-(H3), the cost functional $J_{\varepsilon}(5.3)$ satisfies

$$
\begin{aligned}
& J_{\varepsilon}(t, \eta ; u, v) \geqslant\left(\varepsilon^{-1} \wedge \delta\right) \mathbb{E}_{t}\left\{|X(T)|^{2}+\int_{t}^{T}\left[|u(s)|^{2}+|v(s)|^{2}\right] \mathrm{d} s\right\} \\
& \forall(t, \eta) \in[0, T] \times L_{\mathcal{F}_{t}}^{2}\left(\Omega ; \mathbb{R}^{n}\right),(u, v) \in L_{\mathbb{F}}^{2}\left(t, T ; \mathbb{R}^{m}\right) \times L_{\mathbb{F}}^{2}\left(t, T ; \mathbb{R}^{n}\right) .
\end{aligned}
$$

We now prove the existence of $\alpha_{\varepsilon}$ by contradiction. If not, then for any $\alpha>0$, there exist a $t \in[0, T)$ and an $\Omega_{t} \in \mathcal{F}_{t}$ with $\mathbb{P}\left(\Omega_{t}\right)>0$ such that

$$
\sigma_{\min }\left(P_{\varepsilon}(t, \omega)\right) \leqslant \alpha, \quad \text { a.s. } \omega \in \Omega_{t},
$$

where $\sigma_{\min }\left(P_{\varepsilon}(t, \omega)\right)$ stands for the minimal eigenvalue of the symmetric matrix $P_{\varepsilon}(t, \omega)$. Then we can find an $\mathcal{F}_{t}$-measurable random vector $\eta_{\alpha}$ with $\left|\eta_{\alpha}\right|=1$ such that

$$
\left\langle P_{\varepsilon}(t, \omega) \eta_{\alpha}(\omega), \eta_{\alpha}(\omega)\right\rangle=\sigma_{\min }\left(P_{\varepsilon}(t, \omega)\right) \leqslant \alpha, \quad \text { a.s. } \omega \in \Omega_{t} .
$$

For the fixed $\left(t, \eta_{\alpha}\right)$, by [17], Corollary 5.7 and Theorem 6.7 , there exists a control $\left(u_{\alpha}^{*}, v_{\alpha}^{*}\right) \in L_{\mathbb{F}}^{2}\left(t, T ; \mathbb{R}^{m}\right) \times$ $L_{\mathbb{F}}^{2}\left(t, T ; \mathbb{R}^{n}\right)$ such that

$$
\left\langle P_{\varepsilon}(t) \eta_{\alpha}, \eta_{\alpha}\right\rangle=V_{\varepsilon}\left(t, \eta_{\alpha}\right)=J_{\varepsilon}\left(t, \eta_{\alpha} ; u_{\alpha}^{*}, v_{\alpha}^{*}\right), \quad \text { a.s. }
$$

Combining (5.8) with (5.5), we get

$$
\begin{aligned}
& \mathbb{E}_{t}\left\{\left|X_{\alpha}^{*}(T)\right|^{2}+\int_{t}^{T}\left[\left|u_{\alpha}^{*}(s)\right|^{2}+\left|v_{\alpha}^{*}(s)\right|^{2}\right] \mathrm{d} s\right\} \\
& \quad \leqslant \frac{1}{\left(\varepsilon^{-1} \wedge \delta\right)} J_{\varepsilon}\left(t, \eta_{\alpha} ; u_{\alpha}^{*}, v_{\alpha}^{*}\right)=\frac{1}{\left(\varepsilon^{-1} \wedge \delta\right)}\left\langle P_{\varepsilon}(t) \eta_{\alpha}, \eta_{\alpha}\right\rangle,
\end{aligned}
$$


where $X_{\alpha}^{*}$ is the solution of $(5.2)$ corresponding to $\eta_{\alpha}$ and $\left(u_{\alpha}^{*}, v_{\alpha}^{*}\right)$; that is

$$
\left\{\begin{aligned}
\mathrm{d} X_{\alpha}^{*}(s) & =\left\{A(s) X_{\alpha}^{*}(s)+B(s) u_{\alpha}^{*}(s)+C(s) v_{\alpha}^{*}(s)\right\} \mathrm{d} s+v_{\alpha}^{*}(s) \mathrm{d} W(s), \quad s \in[t, T], \\
X_{\alpha}^{*}(t) & =\eta_{\alpha} .
\end{aligned}\right.
$$

The inequality (5.9), together with (5.7), implies that

$$
\mathbb{E}_{t}\left\{\left|X_{\alpha}^{*}(T)\right|^{2}+\int_{t}^{T}\left[\left|u_{\alpha}^{*}(s)\right|^{2}+\left|v_{\alpha}^{*}(s)\right|^{2}\right] \mathrm{d} s\right\} \leqslant \frac{1}{\left(\varepsilon^{-1} \wedge \delta\right)} \alpha, \quad \text { a.s. on } \Omega_{t} .
$$

Moreover, from (5.10) and the fact $X_{\alpha}^{*}(T)=X_{\alpha}^{*}(T)$, we see that $\left(X_{\alpha}^{*}, v_{\alpha}^{*}\right)$ also satisfies the following BSDE (with $\left(Y_{\alpha}^{*}, Z_{\alpha}^{*}\right)$ being unknown variables):

$$
\left\{\begin{aligned}
\mathrm{d} Y_{\alpha}^{*}(s)= & \left\{A(s) Y_{\alpha}^{*}(s)+B(s) u_{\alpha}^{*}(s)+C(s) Z_{\alpha}^{*}(s)\right\} \mathrm{d} s \\
& +Z_{\alpha}^{*}(s) \mathrm{d} W(s), \quad s \in[t, T] \\
Y_{\alpha}^{*}(T)= & X_{\alpha}^{*}(T)
\end{aligned}\right.
$$

Then, by the estimate (2.2) in Theorem 2.1, there exists a constant $K>0$, independent of $\alpha$ such that

$$
\sup _{s \in[t, T]} \mathbb{E}_{t}\left|X_{\alpha}^{*}(s)\right|^{2}=\sup _{s \in[t, T]} \mathbb{E}_{t}\left|Y_{\alpha}^{*}(s)\right|^{2} \leqslant K \mathbb{E}_{t}\left[\left|X_{\alpha}^{*}(T)\right|^{2}+\int_{t}^{T}\left|u_{\alpha}^{*}(s)\right|^{2} \mathrm{~d} s\right] .
$$

Using (5.13)-(5.11) and the fact that $\left|X_{\alpha}^{*}(t)\right|^{2}=\left|\eta_{\alpha}\right|^{2}=1$, we get

$$
\begin{aligned}
1 & =\left|\eta_{\alpha}\right|^{2}=\left|X_{\alpha}^{*}(t)\right|^{2} \leqslant \sup _{s \in[t, T]} \mathbb{E}_{t}\left|X_{\alpha}^{*}(s)\right|^{2} \\
& \leqslant K \mathbb{E}_{t}\left[\left|X_{\alpha}^{*}(T)\right|^{2}+\int_{t}^{T}\left|u_{\alpha}^{*}(s)\right|^{2} \mathrm{~d} s\right] \leqslant \frac{K}{\left(\varepsilon^{-1} \wedge \delta\right)} \alpha, \quad \text { a.s. on } \quad \Omega_{t},
\end{aligned}
$$

which implies that

$$
1 \leqslant \frac{K}{\left(\varepsilon^{-1} \wedge \delta\right)} \alpha
$$

By taking a small enough $\alpha>0$ such that $\frac{K}{\left(\varepsilon^{-1} \wedge \delta\right)} \alpha<1$, we get the contradiction immediately.

For any $\varepsilon \geqslant 0$, we consider the following controlled FSDE:

$$
\left\{\begin{aligned}
\mathrm{d} X_{\varepsilon}(s) & =\tilde{A}_{\varepsilon}(s) X_{\varepsilon}(s) d s+u(s) \mathrm{d} W(s), \quad s \in[t, T] \\
X_{\varepsilon}(t) & =\eta
\end{aligned}\right.
$$

and cost functional:

$$
\begin{aligned}
\tilde{J}_{\varepsilon}(t, \eta ; u)= & \mathbb{E} \int_{t}^{T}\left[\left\langle\tilde{Q}_{\varepsilon}(s) X_{\varepsilon}(s), X_{\varepsilon}(s)\right\rangle+2\left\langle\tilde{S}(s) X_{\varepsilon}(s), u(s)\right\rangle+\langle\tilde{R}(s) u(s), u(s)\rangle\right] \mathrm{d} s \\
& +\mathbb{E}\left\langle\tilde{G}_{\varepsilon} X_{\varepsilon}(T), X_{\varepsilon}(T)\right\rangle .
\end{aligned}
$$

We introduce the following assumption of the coefficients in (5.16) and weighting matrices in (5.17). 
(H4). For any $\varepsilon \geqslant 0$, the coefficient $\tilde{A}_{\varepsilon}$ and weighting matrices $\tilde{Q}_{\varepsilon}, \tilde{S}, \tilde{R}, \tilde{G}_{\varepsilon}$ satisfy:

$$
\begin{aligned}
& \tilde{A}_{\varepsilon} \in L_{\mathbb{F}}^{\infty}\left(0, T ; \mathbb{R}^{n \times n}\right), \quad \tilde{Q}_{\varepsilon} \in L_{\mathbb{F}}^{\infty}\left(0, T ; \mathbb{S}_{+}^{n}\right), \quad \tilde{S} \in L_{\mathbb{F}}^{\infty}\left(0, T ; \mathbb{R}^{m \times n}\right), \\
& \tilde{R} \in L_{\mathbb{F}}^{\infty}\left(0, T ; \mathbb{S}_{+}^{m}\right), \quad \tilde{G}_{\varepsilon} \in L_{\mathcal{F}_{T}}^{\infty}\left(\Omega ; \mathbb{S}_{+}^{n}\right), \quad \tilde{Q}_{\varepsilon}-\tilde{S}^{\top} \tilde{R}^{-1} \tilde{S} \geqslant 0 .
\end{aligned}
$$

Moreover, there exist two constants $\delta, K>0$, independent of $\varepsilon$ such that

$$
\left|\tilde{A}_{\varepsilon}(s)\right|+\left|\tilde{Q}_{\varepsilon}(s)\right|+\left|\tilde{G}_{\varepsilon}\right| \leqslant K, \quad \tilde{R}(s) \geqslant \delta I_{m}, \quad \text { a.s., a.e. } s \in[0, T] .
$$

With the state equation (5.16) and cost functional (5.17), we consider the following LQ problem:

Problem $\left(\mathbf{S L Q}_{\varepsilon}\right)$. For any given $\varepsilon \geqslant 0$ and $(t, \eta) \in[0, T] \times L_{\mathcal{F}_{t}}^{2}\left(\Omega ; \mathbb{R}^{n}\right)$, find a $u_{\varepsilon}^{*} \in \mathcal{U}[t, T]$ such that

$$
\tilde{J}_{\varepsilon}\left(t, \eta ; u_{\varepsilon}^{*}\right)=\inf _{u \in \mathcal{U}[t, T]} \tilde{J}_{\varepsilon}(t, \eta ; u)=\tilde{V}_{\varepsilon}(t, \eta)
$$

The following result is concerned with the stability of the value functions $\left\{\tilde{V}_{\varepsilon}\right\}_{\varepsilon} \geqslant 0$.

Proposition 5.2. Let (H4) hold. Suppose that

$$
\lim _{\varepsilon \rightarrow 0^{+}}\left[\left|\tilde{A}_{\varepsilon}(s)-\tilde{A}_{0}(s)\right|+\left|\tilde{Q}_{\varepsilon}(s)-\tilde{Q}_{0}(s)\right|+\left|\tilde{G}_{\varepsilon}-\tilde{G}_{0}\right|\right]=0, \quad \text { a.s., a.e. } s \in[0, T] \text {. }
$$

Then for any $(t, \eta) \in[0, T] \times L_{\mathcal{F}_{t}}^{2}\left(\Omega ; \mathbb{R}^{n}\right)$, the following convergence holds:

$$
\lim _{\varepsilon \rightarrow 0^{+}} \tilde{V}_{\varepsilon}(t, \eta)=\tilde{V}_{0}(t, \eta)
$$

Proof. Let $\left(\bar{X}_{\varepsilon}, \bar{Y}_{\varepsilon}, \bar{Z}_{\varepsilon}\right)$ and $\left(\widehat{X}_{\varepsilon}, \widehat{Y}_{\varepsilon}, \widehat{Z}_{\varepsilon}\right)$ be the adapted solutions to the decoupled linear FBSDEs

$$
\left\{\begin{array}{l}
\mathrm{d} \bar{X}_{\varepsilon}(s)=\tilde{A}_{\varepsilon}(s) \bar{X}_{\varepsilon}(s) d s+u(s) \mathrm{d} W(s) \\
\mathrm{d} \bar{Y}_{\varepsilon}(s)=-\left\{\tilde{A}_{\varepsilon}(s)^{\top} \bar{Y}_{\varepsilon}(s)+\tilde{Q}_{\varepsilon}(s) \bar{X}_{\varepsilon}(s)+\tilde{S}(s)^{\top} u(s)\right\} \mathrm{d} s+\bar{Z}_{\varepsilon}(s) \mathrm{d} W(s), \\
\bar{X}_{\varepsilon}(t)=0, \quad \bar{Y}_{\varepsilon}(T)=\tilde{G}_{\varepsilon} \bar{X}_{\varepsilon}(T)
\end{array}\right.
$$

and

$$
\left\{\begin{array}{l}
\mathrm{d} \widehat{X}_{\varepsilon}(s)=\tilde{A}_{\varepsilon}(s) \widehat{X}_{\varepsilon}(s) \mathrm{d} s, \\
\mathrm{~d} \widehat{Y}_{\varepsilon}(s)=-\left\{\tilde{A}_{\varepsilon}(s)^{\top} \widehat{Y}_{\varepsilon}(s)+\tilde{Q}_{\varepsilon}(s) \widehat{X}_{\varepsilon}(s)\right\} \mathrm{d} s+\widehat{Z}_{\varepsilon}(s) \mathrm{d} W(s), \\
\widehat{X}_{\varepsilon}(t)=\eta, \quad \widehat{Y}_{\varepsilon}(T)=\tilde{G}_{\varepsilon} \widehat{X}_{\varepsilon}(T),
\end{array}\right.
$$

respectively. Note that $\left(\bar{X}_{\varepsilon}, \bar{Y}_{\varepsilon}, \bar{Z}_{\varepsilon}\right)$ (respectively, $\left(\widehat{X}_{\varepsilon}, \widehat{Y}_{\varepsilon}, \widehat{Z}_{\varepsilon}\right)$ ) depends linearly on $u$ (respectively, $\left.\eta\right)$. We define two linear operators $\mathcal{N}_{t, \varepsilon}: \mathcal{U}[t, T] \rightarrow \mathcal{U}[t, T]$ and $\mathcal{L}_{t, \varepsilon}: L_{\mathcal{F}_{t}}^{2}\left(\Omega ; \mathbb{R}^{n}\right) \rightarrow \mathcal{U}[t, T]$ as follows:

$$
\begin{aligned}
& {\left[\mathcal{N}_{t, \varepsilon} u\right](s)=\bar{Z}_{\varepsilon}(s)+\tilde{S}(s) \bar{X}_{\varepsilon}(s)+\tilde{R}(s) u(s), \quad s \in[t, T], \quad \forall u \in \mathcal{U}[t, T]} \\
& {\left[\mathcal{L}_{t, \varepsilon} \eta\right](s)=\widehat{Z}_{\varepsilon}(s)+\tilde{S}(s) \widehat{X}_{\varepsilon}(s), \quad s \in[t, T], \quad \forall \eta \in L_{\mathcal{F}_{t}}^{2}\left(\Omega ; \mathbb{R}^{n}\right) .}
\end{aligned}
$$


Since $\tilde{A}_{\varepsilon}, \tilde{Q}_{\varepsilon}, \tilde{G}_{\varepsilon}$ are uniformly bounded by (H4), using the standard estimates of FSDEs ([25], Thm. 6.16, Chap. 1) and BSDEs (Theorem 2.1), it is clear to see that

$$
\left\|\mathcal{N}_{t, \varepsilon} u\right\|^{2} \leqslant K \mathbb{E} \int_{t}^{T}\left[\left|\bar{X}_{\varepsilon}(s)\right|^{2}+\left|\bar{Z}_{\varepsilon}(s)\right|^{2}+|u(s)|^{2}\right] \mathrm{d} s \leqslant K \mathbb{E} \int_{t}^{T}|u(s)|^{2} d s, \quad \forall u \in \mathcal{U}[t, T],
$$

where $K$ is a constant independent of $\varepsilon$ and $u$. Thus the linear operator $\mathcal{N}_{t, \varepsilon}$ is uniformly bounded with respect to $\varepsilon$. Similarly, we can get the uniform boundedness of $\mathcal{L}_{t, \varepsilon}$; that is

$$
\left\|\mathcal{L}_{t, \varepsilon} \eta\right\|^{2} \leqslant K \mathbb{E}|\eta|^{2}, \quad \forall \eta \in L_{\mathcal{F}_{t}}^{2}\left(\Omega ; \mathbb{R}^{n}\right) .
$$

Let $\left(M_{\varepsilon}, H_{\varepsilon}\right)$ be the adapted solution to the following BSDE

$$
\left\{\begin{aligned}
d M_{\varepsilon}(s) & =-\left\{M_{\varepsilon}(s) \tilde{A}_{\varepsilon}(s)+\tilde{A}_{\varepsilon}(s)^{\top} M_{\varepsilon}(s)+\tilde{Q}_{\varepsilon}(s)\right\} \mathrm{d} s+H_{\varepsilon}(s) \mathrm{d} W(s), \\
M_{\varepsilon}(T) & =\tilde{G}_{\varepsilon}
\end{aligned}\right.
$$

By [17], Theorem 3.4, the cost functional $\tilde{J}_{\varepsilon}(t, \eta ; u)$ admits the following representation:

$$
\tilde{J}_{\varepsilon}(t, \eta ; u)=\left\langle\mathcal{N}_{t, \varepsilon} u, u\right\rangle+2\left\langle\mathcal{L}_{t, \varepsilon} \eta, u\right\rangle+\mathbb{E}\left\langle M_{\varepsilon}(t) \eta, \eta\right\rangle .
$$

By (H4)-(5.19), Theorem 2.1, and dominated convergence theorem, we have

$$
\begin{aligned}
& \lim _{\varepsilon \rightarrow 0^{+}} \mathbb{E}\left[\sup _{s \in[t, T]}\left|M_{\varepsilon}(s)-M_{0}(s)\right|^{2}\right] \\
& \quad \leqslant K \lim _{\varepsilon \rightarrow 0^{+}} \mathbb{E} \int_{t}^{T}\left[\left|M_{0}(s)\right|^{2}\left|\tilde{A}_{\varepsilon}(s)-\tilde{A}_{0}(s)\right|^{2}+\left|\tilde{Q}_{\varepsilon}(s)-\tilde{Q}_{0}(s)\right|^{2}\right] \mathrm{d} s=0 .
\end{aligned}
$$

Note that by (H4) and [17], Proposition 2.2, $M_{\varepsilon}$ is uniformly bounded with respect to $\varepsilon$. Thus by dominated convergence theorem again, the above implies that

$$
\lim _{\varepsilon \rightarrow 0^{+}} \mathbb{E}\left\langle M_{\varepsilon}(t) \eta, \eta\right\rangle=\mathbb{E}\left\langle M_{0}(t) \eta, \eta\right\rangle
$$

For any given $u \in \mathcal{U}[t, T]$, by (5.19) and the standard estimates of SDEs and BSDEs, we have

$$
\lim _{\varepsilon \rightarrow 0^{+}} \mathbb{E}\left[\sup _{s \in[t, T]}\left|\bar{X}_{\varepsilon}(s)-\bar{X}_{0}(s)\right|^{2}\right] \leqslant K \lim _{\varepsilon \rightarrow 0^{+}} \mathbb{E} \int_{t}^{T}\left|\tilde{A}_{\varepsilon}(s) \bar{X}_{0}(s)-\tilde{A}_{0}(s) \bar{X}_{0}(s)\right|^{2} \mathrm{~d} s=0,
$$

and

$$
\begin{aligned}
& \lim _{\varepsilon \rightarrow 0^{+}} \mathbb{E}\left[\sup _{s \in[t, T]}\left|\bar{Y}_{\varepsilon}(s)-\bar{Y}_{0}(s)\right|^{2}\right]+\lim _{\varepsilon \rightarrow 0^{+}} \mathbb{E} \int_{t}^{T}\left|\bar{Z}_{\varepsilon}(s)-\bar{Z}_{0}(s)\right|^{2} \mathrm{~d} s \\
& \leqslant K \lim _{\varepsilon \rightarrow 0^{+}} \mathbb{E} \int_{t}^{T}\left|\tilde{A}_{\varepsilon}(s)^{\top} \bar{Y}_{0}(s)+\tilde{Q}_{\varepsilon}(s) \bar{X}_{\varepsilon}(s)-\tilde{A}_{0}(s)^{\top} \bar{Y}_{0}(s)-\tilde{Q}_{0}(s) \bar{X}_{0}(s)\right|^{2} \mathrm{~d} s \\
& \quad+K \lim _{\varepsilon \rightarrow 0^{+}} \mathbb{E}\left|\tilde{G}_{\varepsilon} \tilde{X}_{\varepsilon}(T)-\tilde{G}_{0} \tilde{X}_{0}(T)\right|^{2} \\
&=0 .
\end{aligned}
$$


Combining (5.30) with (5.31), by the definition (5.23) of $\mathcal{N}_{t, \varepsilon}$, we get

$$
\lim _{\varepsilon \rightarrow 0^{+}}\left\|\mathcal{N}_{t, \varepsilon} u-\mathcal{N}_{t, 0} u\right\|=0, \quad \forall u \in \mathcal{U}[t, T]
$$

Similarly, we have

$$
\lim _{\varepsilon \rightarrow 0^{+}}\left\|\mathcal{L}_{t, \varepsilon} \eta-\mathcal{L}_{t, 0} \eta\right\|=0, \quad \forall \eta \in L_{\mathcal{F}_{t}}^{2}\left(\Omega ; \mathbb{R}^{n}\right)
$$

For any $u \in \mathcal{U}[t, T]$, by the representation (5.27) of the cost functional and (H4), we have

$$
\begin{aligned}
\left\langle\mathcal{N}_{t, \varepsilon} u, u\right\rangle= & \tilde{J}_{\varepsilon}(t, 0 ; u) \\
= & \mathbb{E} \int_{t}^{T}\left[\left\langle\tilde{Q}_{\varepsilon}(s) \bar{X}_{\varepsilon}(s), \bar{X}_{\varepsilon}(s)\right\rangle+2\left\langle\tilde{S}(s) \bar{X}_{\varepsilon}(s), u(s)\right\rangle+\langle\tilde{R}(s) u(s), u(s)\rangle\right] \mathrm{d} s \\
& +\mathbb{E}\left\langle\tilde{G}_{\varepsilon} \bar{X}_{\varepsilon}(T), \bar{X}_{\varepsilon}(T)\right\rangle \\
\geqslant & \mathbb{E} \int_{t}^{T}\left[\left\langle\tilde{Q}_{\varepsilon}(s) \bar{X}_{\varepsilon}(s), \bar{X}_{\varepsilon}(s)\right\rangle+2\left\langle\tilde{S}(s) \bar{X}_{\varepsilon}(s), u(s)\right\rangle+\langle\tilde{R}(s) u(s), u(s)\rangle\right] \mathrm{d} s \\
= & \mathbb{E} \int_{t}^{T}\left[\left\langle\left(\tilde{Q}_{\varepsilon}-\tilde{S}^{\top} \tilde{R}^{-1} \tilde{S}\right) \bar{X}_{\varepsilon}, \bar{X}_{\varepsilon}\right\rangle+\left\langle\tilde{R}\left(u+\tilde{R}^{-1} \tilde{S} \bar{X}_{\varepsilon}\right), u+\tilde{R}^{-1} \tilde{S} \bar{X}_{\varepsilon}\right\rangle\right] \mathrm{d} s \\
\geqslant & \delta \mathbb{E} \int_{t}^{T}\left|u+\tilde{R}^{-1} \tilde{S} \bar{X}_{\varepsilon}\right|^{2} \mathrm{~d} s .
\end{aligned}
$$

Define a linear operator $\mathcal{T}_{\varepsilon}: \mathcal{U}[t, T] \rightarrow \mathcal{U}[t, T]$ by

$$
\mathcal{T}_{\varepsilon} u=u+\tilde{R}^{-1} \tilde{S} \bar{X}_{\varepsilon}, \quad u \in \mathcal{U}[t, T] .
$$

Then $\mathcal{T}_{\varepsilon}$ is uniformly bounded and bijective, with its inverse $\mathcal{T}_{\varepsilon}^{-1}$ given by

$$
\mathcal{T}_{\varepsilon}^{-1} u=u-\tilde{R}^{-1} \tilde{S} \tilde{X}_{\varepsilon}, \quad u \in \mathcal{U}[t, T],
$$

where $\tilde{X}_{\varepsilon}$ is the solution of

$$
\left\{\begin{aligned}
\mathrm{d} \tilde{X}_{\varepsilon}(s) & =\tilde{A}_{\varepsilon}(s) \tilde{X}_{\varepsilon}(s) d s+\left\{-\tilde{R}^{-1}(s) \tilde{S}(s) \tilde{X}_{\varepsilon}(s)+u(s)\right\} \mathrm{d} W(s) \\
\bar{X}_{\varepsilon}(t) & =0
\end{aligned}\right.
$$

Since $\tilde{A}_{\varepsilon}$ is uniformly bounded, $\left\|\mathcal{T}_{\varepsilon}^{-1}\right\|$ is clearly uniformly bounded with respect to $\varepsilon$. Thus, we have

$$
\mathbb{E} \int_{t}^{T}\left|u+\tilde{R}^{-1} \tilde{S} \bar{X}_{\varepsilon}\right|^{2} \mathrm{~d} s=\left\|\mathcal{T}_{\varepsilon} u\right\|^{2} \geqslant \frac{1}{\left\|\mathcal{T}_{\varepsilon}^{-1}\right\|^{2}}\left\|\mathcal{T}_{\varepsilon}^{-1} T_{\varepsilon} u\right\|^{2}=\frac{1}{\left\|\mathcal{T}_{\varepsilon}^{-1}\right\|^{2}}\|u\|^{2}>\gamma\|u\|^{2},
$$

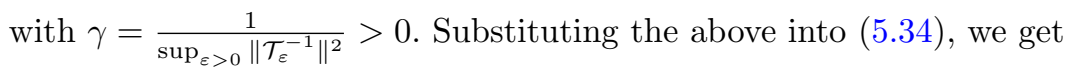

$$
\left\langle\mathcal{N}_{t, \varepsilon} u, u\right\rangle=\tilde{J}_{\varepsilon}(t, 0 ; u) \geqslant \delta \gamma \mathbb{E} \int_{t}^{T}|u(s)|^{2} \mathrm{~d} s
$$


It follows that

$$
\left\|\mathcal{N}_{t, \varepsilon}^{-1}\right\| \leqslant \frac{1}{\delta \gamma}
$$

where $\mathcal{N}_{t, \varepsilon}^{-1}$ is the inverse of $\mathcal{N}_{t, \varepsilon}$. Then by (5.32), we get

$$
\begin{aligned}
\lim _{\varepsilon \rightarrow 0^{+}}\left\|\mathcal{N}_{t, \varepsilon}^{-1} u-\mathcal{N}_{t, 0}^{-1} u\right\| & =\lim _{\varepsilon \rightarrow 0^{+}}\left\|\mathcal{N}_{t, \varepsilon}^{-1} \mathcal{N}_{t, 0} \mathcal{N}_{t, 0}^{-1} u-\mathcal{N}_{t, \varepsilon}^{-1} \mathcal{N}_{t, \varepsilon} \mathcal{N}_{t, 0}^{-1} u\right\| \\
& \leqslant \lim _{\varepsilon \rightarrow 0^{+}}\left\|\mathcal{N}_{t, \varepsilon}^{-1}\right\|\left\|\mathcal{N}_{t, 0} \mathcal{N}_{t, 0}^{-1} u-\mathcal{N}_{t, \varepsilon} \mathcal{N}_{t, 0}^{-1} u\right\| \\
& \leqslant \frac{1}{\delta \gamma} \lim _{\varepsilon \rightarrow 0^{+}}\left\|\mathcal{N}_{t, 0} \mathcal{N}_{t, 0}^{-1} u-\mathcal{N}_{t, \varepsilon} \mathcal{N}_{t, 0}^{-1} u\right\|=0, \quad \forall u \in \mathcal{U}[t, T]
\end{aligned}
$$

By [17], Corollary 3.5, the (unique) optimal control of Problem $\left(\mathrm{SLQ}_{\varepsilon}\right)$ for $\eta$ is given by

$$
u_{\varepsilon}^{*}=-\mathcal{N}_{t, \varepsilon}^{-1} \mathcal{L}_{t, \varepsilon} \eta \text {. }
$$

Substituting the above into (5.27) yields that

$$
\tilde{V}_{\varepsilon}(t, \eta)=\tilde{J}_{\varepsilon}\left(t, \eta ; u_{\varepsilon}^{*}\right)=-\left\langle\mathcal{N}_{t, \varepsilon}^{-1} \mathcal{L}_{t, \varepsilon} \eta, \mathcal{L}_{t, \varepsilon} \eta\right\rangle+\mathbb{E}\left\langle M_{\varepsilon}(t) \eta, \eta\right\rangle, \quad \eta \in L_{\mathcal{F}_{t}}^{2}\left(\Omega ; \mathbb{R}^{n}\right) .
$$

Then combining the above with (5.25)-(5.38), we get

$$
\begin{aligned}
\left|\tilde{V}_{\varepsilon}(t, \eta)-\tilde{V}_{0}(t, \eta)\right|=\mid & -\left\langle\mathcal{N}_{t, \varepsilon}^{-1} \mathcal{L}_{t, \varepsilon} \eta, \mathcal{L}_{t, \varepsilon} \eta\right\rangle+\left\langle\mathcal{N}_{t, 0}^{-1} \mathcal{L}_{t, 0} \eta, \mathcal{L}_{t, 0} \eta\right\rangle+\mathbb{E}\left\langle M_{\varepsilon}(t) \eta, \eta\right\rangle \\
& -\mathbb{E}\left\langle M_{0}(t) \eta, \eta\right\rangle \mid \\
=\mid & -\left\langle\mathcal{N}_{t, \varepsilon}^{-1} \mathcal{L}_{t, \varepsilon} \eta, \mathcal{L}_{t, \varepsilon} \eta-\mathcal{L}_{t, 0} \eta\right\rangle-\left\langle\mathcal{N}_{t, \varepsilon}^{-1}\left(\mathcal{L}_{t, \varepsilon} \eta-\mathcal{L}_{t, 0} \eta\right), \mathcal{L}_{t, 0} \eta\right\rangle \\
& -\left\langle\left(\mathcal{N}_{t, \varepsilon}^{-1}-\mathcal{N}_{t, 0}^{-1}\right) \mathcal{L}_{t, 0} \eta, \mathcal{L}_{t, 0} \eta\right\rangle+\mathbb{E}\left\langle M_{\varepsilon}(t) \eta, \eta\right\rangle-\mathbb{E}\left\langle M_{0}(t) \eta, \eta\right\rangle \mid \\
\leqslant & \left\{\left\|\mathcal{N}_{t, \varepsilon}^{-1}\right\| \times\left\|\mathcal{L}_{t, \varepsilon}\right\| \times\|\eta\| \times\left\|\mathcal{L}_{t, \varepsilon} \eta-\mathcal{L}_{t, 0} \eta\right\|+\left\|\mathcal{N}_{t, \varepsilon}^{-1}\right\| \times\left\|\mathcal{L}_{t, \varepsilon} \eta-\mathcal{L}_{t, 0} \eta\right\|\right. \\
& \left.\times\left\|\mathcal{L}_{t, 0}\right\| \times\|\eta\|+\left\|\mathcal{N}_{t, \varepsilon}^{-1} \mathcal{L}_{t, 0} \eta-\mathcal{N}_{t, 0}^{-1} \mathcal{L}_{t, 0} \eta\right\| \times\left\|\mathcal{L}_{t, 0}\right\| \times\|\eta\|\right\} \\
& +\left|\mathbb{E}\left\langle M_{\varepsilon}(t) \eta, \eta\right\rangle-\mathbb{E}\left\langle M_{0}(t) \eta, \eta\right\rangle\right| \\
\leqslant & K\|\eta\|\left\{\left\|\mathcal{L}_{t, \varepsilon} \eta-\mathcal{L}_{t, 0} \eta\right\|+\left\|\mathcal{N}_{t, \varepsilon}^{-1} \mathcal{L}_{t, 0} \eta-\mathcal{N}_{t, 0}^{-1} \mathcal{L}_{t, 0} \eta\right\|\right\} \\
& +\left|\mathbb{E}\left\langle M_{\varepsilon}(t) \eta, \eta\right\rangle-\mathbb{E}\left\langle M_{0}(t) \eta, \eta\right\rangle\right| .
\end{aligned}
$$

Therefore, by (5.29)-(5.33)-(5.39), we have

$$
\begin{aligned}
\lim _{\varepsilon \rightarrow 0^{+}}\left|\tilde{V}_{\varepsilon}(t, \eta)-\tilde{V}_{0}(t, \eta)\right| \leqslant & K \lim _{\varepsilon \rightarrow 0^{+}}\|\eta\|\left\{\left\|\mathcal{L}_{t, \varepsilon} \eta-\mathcal{L}_{t, 0} \eta\right\|+\left\|\mathcal{N}_{t, \varepsilon}^{-1} \mathcal{L}_{t, 0} \eta-\mathcal{N}_{t, 0}^{-1} \mathcal{L}_{t, 0} \eta\right\|\right\} \\
& +\lim _{\varepsilon \rightarrow 0^{+}}\left|\mathbb{E}\left\langle M_{\varepsilon}(t) \eta, \eta\right\rangle-\mathbb{E}\left\langle M_{0}(t) \eta, \eta\right\rangle\right| \\
& =0, \quad \forall \eta \in L_{\mathcal{F}_{t}}^{2}\left(\Omega ; \mathbb{R}^{n}\right) .
\end{aligned}
$$

We now are ready to state and prove the main result of this section.

Theorem 5.3. Let (H1)-(H2)-(H3) hold. Then Riccati equation (4.1) has a solution $(\Sigma, \Lambda) \in$ $L_{\mathbb{F}}^{\infty}\left(\Omega ; C\left([0, T] ; \mathbb{S}_{+}^{n}\right)\right) \times L_{\mathbb{F}}^{2}\left(0, T ; \mathbb{S}^{n}\right)$. 
Proof. For any $\varepsilon>0$, we consider the following perturbed equation of (4.1):

$$
\left\{\begin{aligned}
\mathrm{d} \Sigma_{\varepsilon}(s)= & {\left[\Sigma_{\varepsilon} A^{\top}+A \Sigma_{\varepsilon}+\Sigma_{\varepsilon} Q \Sigma_{\varepsilon}-B R^{-1} B^{\top}+\Lambda_{\varepsilon} N\left(I_{n}+\Sigma_{\varepsilon} N\right)^{-1} \Lambda_{\varepsilon}\right.} \\
& \left.-C\left(I_{n}+\Sigma_{\varepsilon} N\right)^{-1} \Sigma_{\varepsilon} C^{\top}-C\left(I_{n}+\Sigma_{\varepsilon} N\right)^{-1} \Lambda_{\varepsilon}-\Lambda_{\varepsilon}\left(I_{n}+N \Sigma_{\varepsilon}\right)^{-1} C^{\top}\right] \mathrm{d} s \\
& -\Lambda_{\varepsilon} \mathrm{d} W(s), \quad s \in[0, T], \\
\Sigma_{\varepsilon}(T)= & \varepsilon I_{n} .
\end{aligned}\right.
$$

For the given $\varepsilon>0$, let $\left(P_{\varepsilon}, \Lambda_{\varepsilon}\right) \in L_{\mathbb{F}}^{\infty}\left(\Omega ; C\left([0, T] ; \mathbb{S}_{+}^{n}\right)\right) \times L_{\mathbb{F}}^{2}\left(0, T ; \mathbb{S}^{n}\right)$ be the unique solution of Riccati equation (5.1). By Proposition 5.1, $P_{\varepsilon}$ is uniformly positive definite (for the given $\varepsilon$ ). Hence $P_{\varepsilon}$ is invertible, and its inverse $P_{\varepsilon}^{-1}$ is positive definite and bounded (for the given $\varepsilon$ ). Let

$$
\left(\Sigma_{\varepsilon}, \Lambda_{\varepsilon}\right) \equiv\left(P_{\varepsilon}^{-1}, P_{\varepsilon}^{-1} \Pi_{\varepsilon} P_{\varepsilon}^{-1}\right),
$$

then $\left(\Sigma_{\varepsilon}, \Lambda_{\varepsilon}\right) \in L_{\mathbb{F}}^{\infty}\left(\Omega ; C\left([0, T] ; \mathbb{S}_{+}^{n}\right)\right) \times L_{\mathbb{F}}^{2}\left(0, T ; \mathbb{S}^{n}\right)$. We shall show that $\left(\Sigma_{\varepsilon}, \Lambda_{\varepsilon}\right)$ defined by $(5.42)$ is a solution of (5.41). Using the fact that

$$
0=d\left(\Sigma_{\varepsilon} P_{\varepsilon}\right)=\mathrm{d} \Sigma_{\varepsilon} P_{\varepsilon}+\Sigma_{\varepsilon} \mathrm{d} P_{\varepsilon}+\mathrm{d} \Sigma_{\varepsilon} \mathrm{d} P_{\varepsilon}
$$

we have

$$
\mathrm{d} \Sigma_{\varepsilon}=-\Sigma_{\varepsilon} \mathrm{d} P_{\varepsilon} P_{\varepsilon}^{-1}-\mathrm{d} \Sigma_{\varepsilon} \mathrm{d} P_{\varepsilon} P_{\varepsilon}^{-1}
$$

For convenience, we denote

$$
\mathrm{d} \Sigma_{\varepsilon}(s)=(I) \mathrm{d} s-(I I) \mathrm{d} W(s) .
$$

By (5.43)-(5.1), the diffusion term in (5.44) is given by

$$
(I I)=\Sigma_{\varepsilon} \Pi_{\varepsilon} P_{\varepsilon}^{-1}=P_{\varepsilon}^{-1} \Pi_{\varepsilon} P_{\varepsilon}^{-1}=\Lambda_{\varepsilon}
$$

and then the drift term in (5.44) reads

$$
\begin{aligned}
(I)= & \Sigma_{\varepsilon}\left\{P_{\varepsilon} A+A^{T} P_{\varepsilon}+Q-\left(P_{\varepsilon} C+\Pi_{\varepsilon}\right)\left(N+P_{\varepsilon}\right)^{-1}\left(C^{\top} P_{e}+\Pi_{\varepsilon}\right)\right. \\
& \left.\quad-P_{\varepsilon} B R^{-1} B^{\top} P_{\varepsilon}\right\} P_{\varepsilon}^{-1}+\Sigma_{\varepsilon} \Pi_{\varepsilon} P_{\varepsilon}^{-1} \Pi_{\varepsilon} P_{\varepsilon}^{-1} \\
= & A \Sigma_{\varepsilon}+\Sigma_{\varepsilon} A^{\top}+\Sigma_{\varepsilon} Q \Sigma_{\varepsilon}-C\left(N+P_{\varepsilon}\right)^{-1} C^{\top}-C\left(N+P_{\varepsilon}\right)^{-1} \Pi_{\varepsilon} \Sigma_{\varepsilon} \\
& -\Sigma_{\varepsilon} \Pi_{\varepsilon}\left(N+P_{\varepsilon}\right)^{-1} C^{\top}-\Sigma_{\varepsilon} \Pi_{\varepsilon}\left(N+P_{\varepsilon}\right)^{-1} \Pi_{\varepsilon} \Sigma_{\varepsilon}-B R^{-1} B^{\top}+\Sigma_{\varepsilon} \Pi_{\varepsilon} P_{\varepsilon}^{-1} \Pi_{\varepsilon} \Sigma_{\varepsilon} \\
= & A \Sigma_{\varepsilon}+\Sigma_{\varepsilon} A^{\top}+\Sigma_{\varepsilon} Q \Sigma_{\varepsilon}-C\left(N+P_{\varepsilon}\right)^{-1} C^{\top}-C\left(N+P_{\varepsilon}\right)^{-1} \Pi_{\varepsilon} \Sigma_{\varepsilon} \\
& -\Sigma_{\varepsilon} \Pi_{\varepsilon}\left(N+P_{\varepsilon}\right)^{-1} C^{\top}+\Sigma_{\varepsilon} \Pi_{\varepsilon}\left[P_{\varepsilon}^{-1}\left(N+P_{\varepsilon}\right)-I_{n}\right]\left(N+P_{\varepsilon}\right)^{-1} \Pi_{\varepsilon} \Sigma_{\varepsilon}-B R^{-1} B^{\top} \\
= & A \Sigma_{\varepsilon}+\Sigma_{\varepsilon} A^{\top}+\Sigma_{\varepsilon} Q \Sigma_{\varepsilon}-C\left(N+P_{\varepsilon}\right)^{-1} C^{\top}-C\left(N+P_{\varepsilon}\right)^{-1} \Pi_{\varepsilon} \Sigma_{\varepsilon} \\
& -\Sigma_{\varepsilon} \Pi_{\varepsilon}\left(N+P_{\varepsilon}\right)^{-1} C^{\top}+\Sigma_{\varepsilon} \Pi_{\varepsilon} \Sigma_{\varepsilon} N\left(N+P_{\varepsilon}\right)^{-1} \Pi_{\varepsilon} \Sigma_{\varepsilon}-B R^{-1} B^{\top} .
\end{aligned}
$$

By the definitions of $\Sigma_{\varepsilon}, \Lambda_{\varepsilon}$ and using the facts that

$$
\begin{aligned}
& \left(N+P_{\varepsilon}\right)^{-1}=\left(I_{n}+P_{\varepsilon}^{-1} N\right)^{-1} P_{\varepsilon}^{-1}=\left(I_{n}+\Sigma_{\varepsilon} N\right)^{-1} \Sigma_{\varepsilon} \\
& \left(N+P_{\varepsilon}\right)^{-1}=P_{\varepsilon}^{-1}\left(I_{n}+N P_{\varepsilon}^{-1}\right)^{-1}=\Sigma_{\varepsilon}\left(I_{n}+N \Sigma_{\varepsilon}\right)^{-1}
\end{aligned}
$$


we can rewrite (5.46) as follows:

$$
\begin{aligned}
(I)= & A \Sigma_{\varepsilon}+\Sigma_{\varepsilon} A^{\top}+\Sigma_{\varepsilon} Q \Sigma_{\varepsilon}-C\left(I_{n}+\Sigma_{\varepsilon} N\right)^{-1} \Sigma_{\varepsilon} C^{\top}-C\left(I_{n}+\Sigma_{\varepsilon} N\right)^{-1} \Sigma_{\varepsilon} \Pi_{\varepsilon} \Sigma_{\varepsilon} \\
& -\Sigma_{\varepsilon} \Pi_{\varepsilon} \Sigma_{\varepsilon}\left(I_{n}+N \Sigma_{\varepsilon}\right)^{-1} C^{\top}+\Sigma_{\varepsilon} \Pi_{\varepsilon} \Sigma_{\varepsilon} N\left(I_{n}+\Sigma_{\varepsilon} N\right)^{-1} \Sigma_{\varepsilon} \Pi_{\varepsilon} \Sigma_{\varepsilon}-B R^{-1} B^{\top} \\
= & A \Sigma_{\varepsilon}+\Sigma_{\varepsilon} A^{\top}+\Sigma_{\varepsilon} Q \Sigma_{\varepsilon}-C\left(I_{n}+\Sigma_{\varepsilon} N\right)^{-1} \Sigma_{\varepsilon} C^{\top}-C\left(I_{n}+\Sigma_{\varepsilon} N\right)^{-1} \Lambda_{\varepsilon} \\
& -\Lambda_{\varepsilon}\left(I_{n}+N \Sigma_{\varepsilon}\right)^{-1} C^{\top}+\Lambda_{\varepsilon} N\left(I_{n}+\Sigma_{\varepsilon} N\right)^{-1} \Lambda_{\varepsilon}-B R^{-1} B^{\top} .
\end{aligned}
$$

Note that $\Sigma_{\varepsilon}$ satisfies the terminal condition $\Sigma_{\varepsilon}(T)=P_{\varepsilon}^{-1}(T)=\varepsilon I_{n}$. Substituting (5.47)-(5.45) into (5.44), then it is clearly seen that $\left(\Sigma_{\varepsilon}, \Lambda_{\varepsilon}\right)$ defined by (5.42) satisfies equation (5.41).

By [17], Theorem 5.2 , we have

$$
P_{\varepsilon_{1}} \leqslant P_{\varepsilon_{2}}, \quad \forall 0<\varepsilon_{2} \leqslant \varepsilon_{1}<\infty
$$

Note that for a given $\varepsilon_{0}>0$, Proposition 5.1 shows that there exists a constant $\alpha_{0}>0$ such that

$$
\alpha_{0} I_{n} \leqslant P_{\varepsilon_{0}}
$$

Combining the above with (5.48), we get

$$
\alpha_{0} I_{n} \leqslant P_{\varepsilon_{0}} \leqslant P_{\varepsilon}, \quad \forall 0<\varepsilon \leqslant \varepsilon_{0} .
$$

Since $\Sigma_{\varepsilon}$ is the inverse of $P_{\varepsilon}$, the above implies that

$$
0 \leqslant \Sigma_{\varepsilon} \leqslant \Sigma_{\varepsilon_{0}} \leqslant \frac{1}{\alpha_{o}} I_{n}, \quad \forall 0<\varepsilon \leqslant \varepsilon_{0} .
$$

Then by monotone convergence theorem, there exists a $\Sigma \in L_{\mathbb{F}}^{\infty}\left(0, T ; \mathbb{S}_{+}^{n}\right)$ such that

$$
\lim _{\varepsilon \rightarrow 0^{+}} \Sigma_{\varepsilon}(s)=\Sigma(s), \quad \text { a.s., a.e. } s \in[0, T] \text {. }
$$

We emphasize that in general the above boundedness and monotonicity of $\Sigma_{\varepsilon}$ could not yield

$$
\lim _{\varepsilon \rightarrow 0^{+}} \operatorname{esssup}_{s \in[0, T]}\left|\Sigma_{\varepsilon}(s)-\Sigma(s)\right|=0, \quad \text { a.s. }
$$

Next, we apply Proposition 5.2 to get the existence of the diffusion term $\Lambda$ in (4.1) by introducing the following method of undetermined coefficients. Consider the Riccati equation

$$
\left\{\begin{aligned}
\mathrm{d} \tilde{P}(s)= & -\left\{\tilde{P} \tilde{A}+\tilde{A}^{\top} \tilde{P}+\tilde{Q}-\left(\tilde{S}^{\top}+\tilde{\Pi}\right)(\tilde{R}+\tilde{P})^{-1}(\tilde{S}+\tilde{\Pi})\right\} \mathrm{d} s \\
& +\tilde{\Pi} \mathrm{d} W(s), \quad s \in[0, T] \\
\tilde{P}(T)= & \tilde{G}
\end{aligned}\right.
$$

where

$$
\begin{aligned}
& \tilde{A}=-A^{\top}-Q \Sigma, \quad \tilde{R}=N^{-1}, \quad \tilde{S}=N^{-1} C^{\top}, \quad \tilde{G}=0 \\
& \tilde{Q}=B R^{-1} B^{\top}+\Sigma Q \Sigma+C\left(I_{n}+\Sigma N\right)^{-1} \Sigma C^{\top}+C\left(I_{n}+\Sigma N\right)^{-1} N^{-1} C^{\top} .
\end{aligned}
$$


By (H1)-(H2)-(H3) and the fact that $\Sigma \in L_{\mathbb{F}}^{\infty}\left(0, T ; \mathbb{S}_{+}^{n}\right), \tilde{A}, \tilde{R}, \tilde{Q}, \tilde{S}$ are bounded. Moreover, by (H2)-(H3), we have

$$
\tilde{R}=N^{-1} \geqslant \frac{1}{\lambda} I_{m} \gg 0
$$

and

$$
\begin{aligned}
\tilde{Q}-\tilde{S}^{\top} \tilde{R}^{-1} \tilde{S}= & B R^{-1} B^{\top}+\Sigma Q \Sigma+C\left(I_{n}+\Sigma N\right)^{-1} \Sigma C^{\top} \\
& +C\left(I_{n}+\Sigma N\right)^{-1} N^{-1} C^{\top}-C N^{-1} N N^{-1} C^{\top} \\
= & B R^{-1} B^{\top}+\Sigma Q \Sigma+C N^{-1}\left(N^{-1}+\Sigma\right)^{-1} \Sigma C^{\top} \\
& +C N^{-1}\left(N^{-1}+\Sigma\right)^{-1} N^{-1} C^{\top}-C N^{-1} C^{\top} \\
= & B R^{-1} B^{\top}+\Sigma Q \Sigma+C N^{-1}\left(N^{-1}+\Sigma\right)^{-1}\left(\Sigma+N^{-1}\right) C^{\top}-C N^{-1} C^{\top} \\
= & B R^{-1} B^{\top}+\Sigma Q \Sigma \geqslant 0 .
\end{aligned}
$$

Thus by [16], Proposition 3.5 and [17], Theorem 6.2, Riccati equation (5.54) admits a unique solution $(\tilde{P}, \tilde{\Pi}) \in$ $L_{\mathbb{F}}^{\infty}\left(\Omega ; C\left([0, T] ; \mathbb{S}_{+}^{n}\right)\right) \times L_{\mathbb{F}}^{2}\left(0, T ; \mathbb{S}^{n}\right)$. We claim that if

$$
\tilde{P}=\Sigma,
$$

then $(\tilde{P},-\tilde{\Pi})$ is a solution of (4.1). In fact, with the equality (5.58), equation (5.54) can be rewritten as

$$
\begin{aligned}
\mathrm{d} \tilde{P}(s)=- & \left\{\tilde{P} \tilde{A}+\tilde{A}^{\top} \tilde{P}+\tilde{Q}-\left(\tilde{S}^{\top}+\tilde{\Pi}\right)(\tilde{R}+\tilde{P})^{-1}(\tilde{S}+\tilde{\Pi})\right\} \mathrm{d} s+\tilde{\Pi} \mathrm{d} W(s) \\
=- & \left\{\tilde{P}\left(-A^{\top}-Q \Sigma\right)+(-A-\Sigma Q) \tilde{P}+B R^{-1} B^{\top}+\Sigma Q \Sigma+C\left(I_{n}+\Sigma N\right)^{-1} \Sigma C^{\top}\right. \\
& \left.+C\left(I_{n}+\Sigma N\right)^{-1} N^{-1} C^{\top}-\left(C N^{-1}+\tilde{\Pi}\right)\left(N^{-1}+\tilde{P}\right)^{-1}\left(N^{-1} C^{\top}+\tilde{\Pi}\right)\right\} \mathrm{d} s+\tilde{\Pi} \mathrm{d} W(s) \\
=\{ & A \tilde{P}+\Sigma Q \tilde{P}+\tilde{P} A^{\top}+\tilde{P} Q \Sigma-B R^{-1} B^{\top}-\Sigma Q \Sigma-C\left(I_{n}+\Sigma N\right)^{-1} \Sigma C^{\top} \\
& -C N^{-1}\left(N^{-1}+\Sigma\right)^{-1} N^{-1} C^{\top}+C N^{-1}\left(N^{-1}+\tilde{P}\right)^{-1} N^{-1} C^{\top}+\tilde{\Pi}\left(N^{-1}+\tilde{P}\right)^{-1} \tilde{\Pi} \\
& \left.+\tilde{\Pi}\left(N^{-1}+\tilde{P}\right)^{-1} N^{-1} C^{\top}+C N^{-1}\left(N^{-1}+\tilde{P}\right)^{-1} \tilde{\Pi}\right\} \mathrm{d} s+\tilde{\Pi} \mathrm{d} W(s) \\
=\{ & A \tilde{P}+\tilde{P} A^{\top}+\tilde{P} Q \Sigma-B R^{-1} B^{\top}-C\left(I_{n}+\Sigma N\right)^{-1} \Sigma C^{\top}+\tilde{\Pi}\left(N^{-1}+\tilde{P}\right)^{-1} \tilde{\Pi} \\
& \left.+\tilde{\Pi}\left(N^{-1}+\tilde{P}\right)^{-1} N^{-1} C^{\top}+C N^{-1}\left(N^{-1}+\tilde{P}\right)^{-1} \tilde{\Pi}\right\} \mathrm{d} s+\tilde{\Pi} \mathrm{d} W(s) \\
=\{ & A \tilde{P}+\tilde{P} A^{\top}+\tilde{P} Q \tilde{P}-B R^{-1} B^{\top}-C\left(I_{n}+\tilde{P} N\right)^{-1} \tilde{P} C^{\top}+(-\tilde{\Pi}) N\left(I_{n}+\tilde{P} N\right)^{-1}(-\tilde{\Pi}) \\
& \left.-(-\tilde{\Pi})\left(I_{n}+N \tilde{P}\right)^{-1} C^{\top}-C\left(I_{n}+\tilde{P} N\right)^{-1}(-\tilde{\Pi})\right\} \mathrm{d} s-(-\tilde{\Pi}) \mathrm{d} W(s),
\end{aligned}
$$

which implies that $(\tilde{P},-\tilde{\Pi})$ satisfies Riccati equation (4.1). Thus it suffices to verify that the equality (5.58) holds.

Similar to the arguments in (5.59), we can obtain that $\left(\tilde{P}_{\varepsilon}, \tilde{\Pi}_{\varepsilon}\right) \equiv\left(\Sigma_{\varepsilon},-\Lambda_{\varepsilon}\right)$ satisfies the following Riccati equation

$$
\left\{\begin{aligned}
\mathrm{d} \tilde{P}_{\varepsilon}(s)= & -\left\{\tilde{P}_{\varepsilon} \tilde{A}_{\varepsilon}+\tilde{A}_{\varepsilon}^{\top} \tilde{P}_{\varepsilon}+\tilde{Q}_{\varepsilon}-\left(\tilde{S}^{\top}+\tilde{\Pi}_{\varepsilon}\right)\left(\tilde{R}+\tilde{P}_{\varepsilon}\right)^{-1}\left(\tilde{S}+\tilde{\Pi}_{\varepsilon}\right)\right\} \mathrm{d} s \\
& +\tilde{\Pi}_{\varepsilon} \mathrm{d} W(s), \quad s \in[0, T], \\
\tilde{P}_{\varepsilon}(T)= & \tilde{G}_{\varepsilon},
\end{aligned}\right.
$$


with

$$
\begin{aligned}
& \tilde{A}_{\varepsilon}=-A^{\top}-Q \Sigma_{\varepsilon}, \quad \tilde{R}=N^{-1}, \quad \tilde{S}=N^{-1} C^{\top}, \quad \tilde{G}_{\varepsilon}=\varepsilon I_{n}, \\
& \tilde{Q}_{\varepsilon}=B R^{-1} B^{\top}+\Sigma_{\varepsilon} Q \Sigma_{\varepsilon}+C\left(I_{n}+\Sigma_{\varepsilon} N\right)^{-1} \Sigma_{\varepsilon} C^{\top}+C\left(I_{n}+\Sigma_{\varepsilon} N\right)^{-1} N^{-1} C^{\top} .
\end{aligned}
$$

By (5.51), $\tilde{G}_{\varepsilon}, \tilde{A}_{\varepsilon}, \tilde{Q}_{\varepsilon}$ are uniformly bounded (with respect to $0 \leqslant \varepsilon \leqslant \varepsilon_{0}$ ). Moreover, by (5.52), we have

$$
\begin{gathered}
\lim _{\varepsilon \rightarrow 0^{+}} \tilde{G}_{\varepsilon}=\tilde{G}_{0} \equiv \tilde{G}, \quad \lim _{\varepsilon \rightarrow 0^{+}} \tilde{A}_{\varepsilon}(s)=\tilde{A}_{0}(s) \equiv \tilde{A}(s), \\
\lim _{\varepsilon \rightarrow 0^{+}} \tilde{Q}_{\varepsilon}(s)=\tilde{Q}_{0}(s) \equiv \tilde{Q}(s), \quad \text { a.s., a.e. } s \in[0, T] .
\end{gathered}
$$

Note that $\left(\tilde{P}_{\varepsilon}, \tilde{\Pi}_{\varepsilon}\right)$ is the solution of the corresponding Riccati equation of Problem (SLQ $\tilde{\sigma}_{\varepsilon}$ ), which is defined by (5.18). By [17], Proposition 5.5, the value function $\tilde{V}_{\varepsilon}$ of Problem $\left(\mathrm{SLQ}_{\varepsilon}\right)$ can be given by

$$
\tilde{V}_{\varepsilon}(t, \eta)=\mathbb{E}\left\langle\tilde{P}_{\varepsilon}(t) \eta, \eta\right\rangle, \quad \forall \varepsilon \geqslant 0, \quad \eta \in L_{\mathcal{F}_{t}}^{2}\left(\Omega ; \mathbb{R}^{n}\right) .
$$

Then by Proposition 5.2, the convergence (5.62) implies that

$$
\mathbb{E}\langle\tilde{P}(t) \eta, \eta\rangle=\tilde{V}_{0}(t, \eta)=\lim _{\varepsilon \rightarrow 0^{+}} \tilde{V}_{\varepsilon}(t, \eta)=\lim _{\varepsilon \rightarrow 0^{+}} \mathbb{E}\left\langle\tilde{P}_{\varepsilon}(t) \eta, \eta\right\rangle, \quad \forall \eta \in L_{\mathcal{F}_{t}}^{2}\left(\Omega ; \mathbb{R}^{n}\right) .
$$

Using the fact that $\tilde{P}_{\varepsilon}=\Sigma_{\varepsilon}$, by (5.52)-(5.51) and dominated convergence theorem, we have

$$
\lim _{\varepsilon \rightarrow 0^{+}} \mathbb{E}\left\langle\tilde{P}_{\varepsilon}(t) \eta, \eta\right\rangle=\lim _{\varepsilon \rightarrow 0^{+}} \mathbb{E}\left\langle\Sigma_{\varepsilon}(t) \eta, \eta\right\rangle=\mathbb{E}\langle\Sigma(t) \eta, \eta\rangle, \quad \forall \eta \in L_{\mathcal{F}_{t}}^{2}\left(\Omega ; \mathbb{R}^{n}\right)
$$

Combining (5.63) with (5.64) together, we get

$$
\mathbb{E}\langle\tilde{P}(t) \eta, \eta\rangle=\mathbb{E}\langle\Sigma(t) \eta, \eta\rangle, \quad \forall \eta \in L_{\mathcal{F}_{t}}^{2}\left(\Omega ; \mathbb{R}^{n}\right) .
$$

It follows that

$$
\tilde{P}(t)=\Sigma(t), \quad \text { a.s., } \quad \text { a.e. } t \in[0, T] .
$$

Thus the equality (5.58) holds and the proof is completed.

Remark 5.4. From the proof of Theorem 5.3, we see that if Riccati equation (4.1) has a solution $(\Sigma, \Lambda)$, it can be rewritten as a Riccati equation associated with some forward LQ problem (see (5.54) with $\tilde{P}=\Sigma$ ). Noticing this fact, to prove the solvability of Riccati equation (4.1), we only need to show (5.58) holds. Since the coefficients of (5.54) depend on $\Sigma$, which is a undetermined variable, we would like to call the above arguments a method of undetermined coefficients.

With a solved solution $(\Sigma, \Lambda)$ of Riccati equation (4.1), the following result shows that the decoupled system of BSDE (4.2) and FSDE (4.3) is uniquely solvable.

Theorem 5.5. Let $(\mathrm{H} 1)-(\mathrm{H} 2)-(\mathrm{H} 3)$ hold and $(\Sigma, \Lambda) \in L_{\mathbb{F}}^{\infty}\left(\Omega ; C\left([0, T] ; \mathbb{S}_{+}^{n}\right)\right) \times L_{\mathbb{F}}^{2}\left(0, T ; \mathbb{S}^{n}\right)$ be a solution of Riccati equation (4.1). Then for any $\xi \in L_{\mathcal{F}_{T}}^{2}\left(\Omega ; \mathbb{R}^{n}\right)$, the decoupled system of BSDE (4.2) and FSDE (4.3) admits a unique solution $(\varphi, \beta, X) \in L_{\mathbb{F}}^{2}\left(\Omega ; C\left([t, T] ; \mathbb{R}^{n}\right)\right) \times L_{\mathbb{F}}^{1}\left(\Omega ; L^{2}\left(t, T ; \mathbb{R}^{n}\right)\right) \times L_{\mathbb{F}}^{2}\left(\Omega ; C\left([t, T] ; \mathbb{R}^{n}\right)\right)$. 
Proof. Let $\left(X^{*}, Y^{*}, Z^{*}\right)$ be the unique solution of the coupled FBSDE (3.12) and $(\Sigma, \Lambda)$ be a solution of Riccati equation (4.1). Define

$$
\varphi^{*} \equiv-\Sigma X^{*}-Y^{*}, \quad \beta^{*} \equiv\left(\Sigma N+I_{n}\right) Z^{*}-\Sigma C^{\top} X^{*}-\Lambda X^{*} .
$$

It is clear to see that $\left(\varphi^{*}, \beta^{*}\right) \in L_{\mathbb{F}}^{2}\left(\Omega ; C\left([t, T] ; \mathbb{R}^{n}\right)\right) \times L_{\mathbb{F}}^{1}\left(\Omega ; L^{2}\left(t, T ; \mathbb{R}^{n}\right)\right)$. We shall show that $\left(\varphi^{*}, \beta^{*}\right)$ is a solution of BSDE (4.2). In fact, by applying Itô's formula to $s \mapsto \varphi^{*}(s) \equiv-\Sigma(s) X^{*}(s)-Y^{*}(s)$, we have

$$
\begin{aligned}
& \mathrm{d} \varphi^{*}(s) \equiv d\left[-\Sigma(s) X^{*}(s)-Y^{*}(s)\right] \\
& =\left\{-\left[\Sigma A^{\top}+A \Sigma+\Sigma Q \Sigma-B R^{-1} B^{\top}+\Lambda N(\Sigma N+I)^{-1} \Lambda-C(I+\Sigma N)^{-1} \Sigma C^{\top}\right.\right. \\
& \left.-C(\Sigma N+I)^{-1} \Lambda-\Lambda(N \Sigma+I)^{-1} C^{\top}\right] X^{*}-\Sigma\left[-A^{\top} X^{*}+Q Y^{*}\right] \\
& \left.+\Lambda\left[-C^{\top} X^{*}+N Z^{*}\right]-\left[A Y^{*}+B R^{-1} B^{\top} X^{*}+C Z^{*}\right]\right\} \mathrm{d} s \\
& +\left\{\Lambda X^{*}-\Sigma\left[-C^{\top} X^{*}+N Z^{*}\right]-Z^{*}\right\} \mathrm{d} W(s) \\
& =\left\{-A\left(\Sigma X^{*}+Y^{*}\right)-\Sigma Q\left(\Sigma X^{*}+Y^{*}\right)-\Lambda N(\Sigma N+I)^{-1} \Lambda X^{*}+\Lambda(N \Sigma+I)^{-1} C^{\top} X^{*}\right. \\
& \left.-\Lambda C^{\top} X^{*}+\Lambda N Z^{*}+C(I+\Sigma N)^{-1} \Sigma C^{\top} X^{*}+C(\Sigma N+I)^{-1} \Lambda X^{*}-C Z^{*}\right\} \mathrm{d} s \\
& -\left\{\left(\Sigma N+I_{n}\right) Z^{*}-\Sigma C^{\top} X^{*}-\Lambda X^{*}\right\} \mathrm{d} W(s) \\
& =\left\{-(A+\Sigma Q)\left(\Sigma X^{*}+Y^{*}\right)+\Lambda N(\Sigma N+I)^{-1}\left[\left(\Sigma N+I_{n}\right) Z^{*}-\Sigma C^{\top} X^{*}-\Lambda X^{*}\right]\right. \\
& \left.-C(\Sigma N+I)^{-1}\left[\left(\Sigma N+I_{n}\right) Z^{*}-\Sigma C^{\top} X^{*}-\Lambda X^{*}\right]\right\} \mathrm{d} s \\
& -\left\{\left(\Sigma N+I_{n}\right) Z^{*}-\Sigma C^{\top} X^{*}-\Lambda X^{*}\right\} \mathrm{d} W(s), \quad s \in[t, T] .
\end{aligned}
$$

With the definition $(5.65)$ of $\left(\varphi^{*}, \beta^{*}\right)$, the above can be rewritten as

$$
\begin{aligned}
\mathrm{d} \varphi^{*}(s)=\{ & \left.(A+\Sigma Q) \varphi^{*}(s)+\Lambda N(\Sigma N+I)^{-1} \beta^{*}(s)-C(\Sigma N+I)^{-1} \beta^{*}(s)\right\} \mathrm{d} s \\
& -\beta^{*}(s) \mathrm{d} W(s), \quad s \in[t, T] .
\end{aligned}
$$

Since $\varphi^{*}$ also satisfies the terminal condition:

$$
\varphi^{*}(T)=-\Sigma(T) X^{*}(T)-Y^{*}(T)=-Y^{*}(T)=-\xi,
$$

$\left(\varphi^{*}, \beta^{*}\right)$ is a solution of (4.2). Moreover, (5.65) implies that

$$
Y^{*}=-\Sigma X^{*}-\varphi^{*}, \quad Z^{*}=\left(I_{n}+\Sigma N\right)^{-1}\left[\beta^{*}+\Sigma C^{\top} X^{*}+\Lambda X^{*}\right] .
$$

Substituting the above into the FSDE in (3.12), we have

$$
\begin{aligned}
d X^{*}(s)=- & \left\{\left(A^{\top}+Q \Sigma\right) X^{*}(s)+Q \varphi^{*}\right\} \mathrm{d} s+\left\{-C^{\top} X^{*}(s)+N\left(I_{n}+\Sigma N\right)^{-1} \beta^{*}\right. \\
& \left.+N\left(I_{n}+\Sigma N\right)^{-1}\left(\Lambda+\Sigma C^{\top}\right) X^{*}(s)\right\} \mathrm{d} W(s), \quad s \in[t, T] .
\end{aligned}
$$

Note that

$$
Y^{*}(t)=-\Sigma(t) X^{*}(t)-\varphi^{*}(t)=-\Sigma(t) G_{t} Y^{*}(t)-\varphi^{*}(t),
$$


we get

$$
Y^{*}(t)=-\left(I_{n}+\Sigma(t) G_{t}\right)^{-1} \varphi^{*}(t)
$$

which implies that

$$
X^{*}(t)=G_{t} Y^{*}(t)=-G_{t}\left(I_{n}+\Sigma(t) G_{t}\right)^{-1} \varphi^{*}(t)=-\left(I_{n}+G_{t} \Sigma(t)\right)^{-1} G_{t} \varphi^{*}(t) .
$$

Combining the above with (5.68), $X^{*}$ satisfies the $\operatorname{FSDE}(4.3)$ with $(\varphi, \beta)$ given by $\left(\varphi^{*}, \beta^{*}\right)$.

Let $(\varphi, \beta) \in L_{\mathbb{F}}^{2}\left(\Omega ; C\left([t, T] ; \mathbb{R}^{n}\right)\right) \times L_{\mathbb{F}}^{1}\left(\Omega ; L^{2}\left(t, T ; \mathbb{R}^{n}\right)\right)$ be any solution of BSDE (4.2) and $X \in$ $L_{\mathbb{F}}^{2}\left(\Omega ; C\left([t, T] ; \mathbb{R}^{n}\right)\right)$ be any solution of $\operatorname{FSDE}(4.3)$ corresponding to the given $(\varphi, \beta)$. Let

$$
Y \equiv-\Sigma X-\varphi, \quad Z \equiv\left(I_{n}+\Sigma N\right)^{-1}\left[\Sigma C^{\top} X+\beta+\Lambda X\right]
$$

By Theorem 4.1, $(X, Y, Z)$ is the unique solution of FBSDE (3.1). Then the uniqueness of $(\varphi, \beta, X)$ follows from the uniqueness of the adapted solutions to (3.1) immediately.

Remark 5.6. By Theorem 5.3 and Theorem 5.5, the solvabilities of Riccati equation (4.1), BSDE (4.2) and SDE (4.3) are established. Then a complete and explicit representation (4.7) for the optimal control of Problem (BLQ) is obtained, via the solutions to Riccati equation (4.1), BSDE (4.2) and SDE (4.3).

Acknowledgements. The authors would like to thank the associate editor and the anonymous referees for their suggestive comments, which lead to this improved version of the paper.

\section{REFERENCES}

[1] M. Ait Rami, J.B. Moore and X.Y. Zhou, Indefinite stochastic linear quadratic control and generalized differential Riccati equation. SIAM J. Control Optim. 40 (2001) 1296-1311.

[2] X. Bi, J. Sun and J. Xiong, Optimal control for controllable stochastic linear systems. ESAIM: COCV 26 (2020) 98.

[3] J.M. Bismut, Linear quadratic optimal stochastic control with random coefficients. SIAM J. Control Optim. 14 (1976) 419-444.

[4] S. Chen, X. Li and X.Y. Zhou, Stochastic linear quadratic regulators with indefinite control weight costs. SIAM J. Control Optim. 36 (1998) 1685-1702.

[5] S. Chen and J. Yong, Stochastic linear quadratic optimal control problems. Appl. Math. Optim. 43 (2001) $21-45$.

[6] S. Chen and X.Y. Zhou, Stochastic linear quadratic regulators with indefinite control weight costs. II. SIAM J. Control Optim. 39 (2000) 1065-1081.

[7] K. Du, J. Huang and Z. Wu, Linear quadratic mean-field-game of backward stochastic differential systems. Math. Control Relat. Fields 8 (2018) 653-678.

[8] J. Huang, S. Wang and Z. Wu, Backward mean-field linear-quadratic-Gaussian (LQG) games: full and partial information. IEEE Trans. Automat. Control 61 (2016) 3784-3796.

[9] M. Kohlmann and S. Tang, Multidimensional backward stochastic Riccati equations and applications. SIAM J. Control Optim. 41 (2003) 1696-1721.

[10] X. Li, J. Sun and J. Xiong, Linear quadratic optimal control problems for mean-field backward stochastic differential equations. Appl. Math. Optim. 80 (2019) 223-250.

[11] A.E.B. Lim, Quadratic hedging and mean-variance portfolio selection with random parameters in an incomplete market. Math. Oper. Res. 29 (2004) 132-161.

[12] A.E.B. Lim and X.Y. Zhou, Linear-quadratic control of backward stochastic differential equations. SIAM J. Control Optim. 40 (2001) 450-474.

[13] J. Ma and J. Yong, Forward-Backward Stochastic Differential Equations and Their Applications. Vol. 1702 of Lecture Notes Math. Springer-Verlag, Berlin (1999).

[14] S. Peng, Open problems on backward stochastic differential equations, in Control of Distributed Parameter and Stochastic Systems. edited by S. Chen, X. Li, J. Yong and X.Y. Zhou. Springer, Boston, MA (1999) 265-273.

[15] N. EL Karoui, S. Peng and M.C. Quenez, Backward stochastic differential equations in finance. Math. Finance 7 (1997) 1-71.

[16] J. Sun, X. Li and J. Yong, Open-loop and closed-loop solvabilities for stochastic linear quadratic optimal control problems. SIAM J. Control Optim. 54 (2016) 2274-2308.

[17] J. Sun, J. Xiong and J. Yong, Stochastic linear-quadratic optimal control problems with random coefficients: Closed-loop representation of open-loop optimal controls. Ann. Appl. Probab. 31 (2021) 460-499. 
[18] J. Sun and J. Yong, Stochastic Linear-Quadratic Optimal Control Theory: Open-Loop and Closed-Loop Solutions. Springer Briefs in Mathematics (2020).

[19] S. Tang, General linear quadratic optimal stochastic control problems with random coefficients: linear stochastic Hamilton systems and backward stochastic Riccati equations. SIAM J. Control Optim. 42 (2003) 53-75.

[20] S. Tang, Dynamic programming for general linear quadratic optimal stochastic control with random coefficients. SIAM J. Control Optim. 53 (2015) 1082-1106.

[21] H. Wang, J. Sun and J. Yong, Weak closed-loop solvability of stochastic linear-quadratic optimal control problems. Discete Contin. Dyn. Syst. 39 (2019) 2785-2805.

[22] G. Wang, H. Xiao and J. Xiong, A kind of LQ non-zero sum differential game of backward stochastic differential equation with asymmetric information. Automatica 97 (2018) 346-352.

[23] W.M. Wonham, On a matrix Riccati equation of stochastic control. SIAM J. Control 6 (1968) 681-697.

[24] J. Yong, A leader-follower stochastic linear quadratic differential game. SIAM J. Control Optim. 41 (2002) $1015-1041$.

[25] J. Yong and X.Y. Zhou, Stochastic Controls: Hamiltonian Systems and HJB Equations. Springer-Verlag, New York (1999). 